\title{
Dynamic Inventory Control with Limited Capital and Short-Term Financing
}

\author{
Xiting Gong, ${ }^{1}$ Xiuli Chao, ${ }^{2}$ David Simchi-Levi ${ }^{3}$ \\ ${ }^{1}$ Department of Systems Engineering and Engineering Management, The Chinese University of Hong Kong, Shatin, New \\ Territories, Hong Kong \\ ${ }^{2}$ Department of Industrial and Operations Engineering, University of Michigan, Ann Arbor, Michigan 48109 \\ ${ }^{3}$ Engineering Systems Division and Department of Civil and Environmental Engineering, \\ Massachusetts Institute of Technology, Cambridge, Massachusetts 02139
}

\begin{abstract}
Received 18 September 2012; revised 28 July 2013 and 18 January 2014; accepted 18 January 2014
DOI 10.1002/nav.21576

Published online 18 February 2014 in Wiley Online Library (wileyonlinelibrary.com).
\end{abstract}

\begin{abstract}
For most firms, especially the small- and medium-sized ones, the operational decisions are affected by their internal capital and ability to obtain external capital. However, the majority of the literature on dynamic inventory control ignores the firm's financial status and financing issues. An important question that arises is: what are the optimal inventory and financing policies for firms with limited internal capital and limited access to external capital? In this article, we study a dynamic inventory control problem where a capital-constrained firm periodically purchases a product from a supplier and sells it to a market with random demands. In each period, the firm can use its own capital and/or borrow a short-term loan to purchase the product, with the interest rate being nondecreasing in the loan size. The objective is to maximize the firm's expected terminal wealth at the end of the planning horizon. We show that the optimal inventory policy in each period is an equity-level-dependent base-stock policy, where the equity level is the sum of the firm's capital level and the value of its on-hand inventory evaluated at the purchasing cost; and the structure of the optimal policy can be characterized by four intervals of the equity level. Our results shed light on the dynamic inventory control for firms with limited capital and short-term financing capabilities. (C) 2014 Wiley Periodicals, Inc. Naval Research Logistics 61: 184-201, 2014
\end{abstract}

Keywords: inventory control; financial constraint; short-term financing; dynamic programming; optimal policy

\section{INTRODUCTION}

The majority of the literature on dynamic inventory control ignores the financial status of a firm and assumes that it always has enough capital to implement any operational decisions (see, e.g., Zipkin [23]). Such ignorance may be justified by the seminal paper of Modigliani and Miller [15], which implies that a firm's operational and financial decisions can be made separately in a perfect capital market. In practice, however, most firms (especially the start-up, small-, and medium-sized ones) do not operate in perfect capital markets, and their operational decisions are affected by their internal capital and ability to obtain external capital such as bank loans, equity, and venture capital investment. Indeed, according to a report from the Federal Reserve Bank of New York, for firms with limited access to capital markets, internal fund is a significant predicator of inventory investment

Correspondence to: X. Gong (xtgong@se.cuhk.edu.hk)
(Zakrajšek [22]). Thus, an important question that arises is: what are the optimal inventory and financing policies for the firms with limited internal capital and limited access to external capital? For instance, it is well-known that a base-stock policy is optimal for the classic periodic-review inventory system, so how does the optimal inventory policy change if the firm has limited capital and can only obtain short-term loans?

In this article, we address this question by studying a dynamic inventory control problem where a capitalconstrained firm periodically purchases a product from a supplier and sells it to a market with random demands. In each period, the firm can use its own capital and/or borrow a short-term loan from a lender to purchase the product, with the total loan interest being an increasing and convex function of the loan size. The firm can also deposit its extra capital into a savings account with a fixed interest rate. The random demand is realized during the period, and the sales revenue is then collected. At the end of the period, the firm's capital is 
updated after receiving its deposited capital and interest from the savings account and paying the principal and interest of the short-term loan. Meanwhile, unsold product is carried to the next period and it is salvaged at the end of the planning horizon; and any unmet demand is lost. The firm's objective is to maximize its expected terminal wealth (capital) at the end of the planning horizon.

We formulate the firm's problem as a stochastic dynamic program with two state variables: the firm's inventory level and capital level at the beginning of each period. By studying the structural properties of the dynamic program, we show that an equity-level-dependent base-stock inventory policy is optimal in each period, where the equity level is the sum of the firm's capital level and the value of its on-hand inventory evaluated at the purchasing cost. This result is consistent with the finding in Zakrajšek [22] that "the observed volatility in aggregate retail inventory ... is due to fluctuations in internal funds". We further study the structure of the firm's optimal base-stock policy, and show that it can be characterized by dividing the equity level into four different intervals. In one of these intervals, the firm does not order regardless of its inventory; and in the second one, the firm has a constant optimal base-stock level and only uses its own capital for replenishment. In the third interval, the optimal base-stock level equals the amount of products the equity can purchase, and in this case, the firm simply uses up its capital for replenishment and does not borrow from the lender. In the fourth and last interval, the optimal base-stock level is a capital-dependent curve and may not be even monotone in the equity level. In this interval, some partial structural results are obtained. We also extend the base model to the case with physical holding cost, the case with reorganization bankruptcy, and the case with fixed borrowing cost. We show that most of our results continue to hold under the first two extensions; while for the third extension, the problem is much more complicated and we characterize the optimal policy only for the last-period problem. Our results shed light on the optimal dynamic inventory control for firms with limited capital and short-term financing.

This work is mainly related to the literature on inventory systems with financial considerations. Most of the existing studies are based on single-period models, in which decision makers have only one chance to make operational and financial decisions under financial constraints. Some of these models focus on a single firm's optimal decisions. For example, Xu and Birge $[18,20]$ study joint production and financing decisions of a capital-constrained firm in the presence of demand uncertainty, market imperfections, and managerial incentives; and Babich et al. [2] study the joint procurement and financing decisions of a capital-constrained firm, facing either an uncertain demand or an uncertain supply. There are a number of research papers on single-period models studying the effects of financial constraints and financing sources with strategic interactions in supply chains. For example, Buzacott and Zhang [4] incorporate asset-based financing into production decisions and analyze a Stackelberg game between a bank and a retailer; and Dada and Hu [6] study a capital-constrained newsverdor's optimal borrowing amount when a bank determines the interest rate to maximize its own profits. Recently, Kouvelis and Zhao [13] study a Stackelberg game between a supplier and a retailer when the retailer faces bankruptcy risk if it borrows from a bank; and Yang and Birge [21] analyze the effects of trade credit in supply chains with costs of financial distress. The main difference between these models and ours is that we study a dynamic inventory control model where a single firm makes multiple operational and financial decisions during the planning horizon.

In contrast to many studies on single-period models, only a few studies have considered dynamic inventory models with financial considerations. Two of these studies focus on the self-financing firms which solely rely on their internal capital (and sales revenue) to operate. Archibald et al. [1] study the optimal ordering policy of a start-up firm with the objective to maximize its probability of long-term survival. Chao et al. [5] study the optimal inventory policy of a self-financing firm to maximize its expected terminal wealth and show that the optimal policy is a modified base-stock policy (i.e., there exists a base-stock level in each period, and the optimal policy aims to raise the inventory to this level subject to the firm's capital). The other studies incorporate one or more external financing sources (e.g., bank loans, debt, and capital subscription) into the dynamic models. Babich and Sobel [3] incorporate short-term loans with a fixed interest rate and study a firm's optimal operational and financial decisions to maximize its expected discounted proceeds from an initial public offering. Xu and Birge [19] propose a finite-horizon integrated planning model for a firm, which can both borrow short-term loans and issue equity, to maximize the expected discounted value of net cash flow to the firm's shareholders. Their model is an integer stochastic program with nonlinear constraints; and the authors develop an efficient algorithm to solve it numerically. With a similar objective to $\mathrm{Xu}$ and Birge [19] and under the assumption that the firm continues to operate but pays a default penalty when it goes bankrupt, $\mathrm{Hu}$ and Sobel [10] study a multi-echelon inventory model and show that echelon base-stock policies are in general not optimal with financial constraints; and Li et al. [14] study the optimal inventory and financial policies for a single-echelon model, and they compare their model with a decentralized system where operational and financial decisions are made separately. Under the alternative assumption that the firm stops operations if it goes bankrupt, Hu et al. [11] study a model with coordinated inventory and financial decisions and analyze it against the corresponding model with decentralized decisions. The reader is referred to Hu et al. [9] for more discussions along the line of $\mathrm{Li}$ et al. [14] and $\mathrm{Hu}$ et al. [11]. 
The papers that are most closely related to ours are Chao et al. [5] and Li et al. [14], but there are significant differences. First, compared with Chao et al. [5] which focuses on a self-financing firm, our study extends this work by allowing short-term financing. We remark that the inclusion of shortterm financing has important economic implications, as there are many financing companies that provide short-term loans to small companies. For example, American Microloan is such a company that provides cash advances and quick loans to small businesses in the form of short-term working capital. ${ }^{1}$ If firms ignore such financing opportunities and solely rely on their own capital, they may lose potential profits and development opportunities. On the other hand, the inclusion of short-term financing also brings significant challenges on the firm's operational and financing decisions. When the firm is self-financed, its optimal policy has a very simple modified base-stock type, as afore mentioned. In contrast, when short-term financing is an option for the firm, its optimal policy becomes much more complicated even if the loan interest rate is a constant. Technically speaking, the simple policy structure in Chao et al. [5] is due to the fact that the bivariate value function can be fully decoupled into two univariate functions; whereas in our model, the value function can only be decoupled within a certain range, which results in more challenging analysis and more complicated policy structure. In addition, while Chao et al. [5] are unable to deal with features such as physical holding cost and bankruptcy cost, we can incorporate them into our model and show that they do not significantly change our structural results. We note that Shi et al. [17] also analyze a dynamic inventory control problem under cash-flow constraints, but they focus on developing simple myopic heuristic policies and bounds for the control parameters; their demand process, however, is allowed to be nonstationary.

Second, compared with Li et al. [14] in which the firm's objective is to maximize the expected present value of dividends net of capital subscription, the objective in our study is to maximize the firm's expected terminal wealth. Under Li et al. [14]'s setting, a salient result is that the firm's problem can be simplified to have a single-state variable (i.e., the inventory level). The reason is that, as subscribing capital and issuing dividend have the same marginal value in Li et al. [14], the firm's capital has a constant marginal value in each period, thus the dynamic program can be formulated to include the inventory level as the single state variable. In contrast, as in our setting the deposit and borrowing have different marginal values, the firm's capital does not have a constant marginal value, thus both the inventory level and the capital level must be included as state variables, resulting in a multidimensional state space. Because of this key difference, our analysis and results are quite different from Li et al. [14]'s.

\footnotetext{
${ }^{1}$ Refer to http://www.americanmicroloan.com/.
}

The rest of this article is organized as follows. In Section 2 , we present the model and its mathematical formulation in detail. In Section 3, we study the structural properties of the model and the firm's optimal control policy. In Section 4 , we extend the base model to the case with physical holding cost, the case with reorganization bankruptcy, and the case with fixed borrowing cost. We conclude the article in Section 5 with some possible future directions. All technical proofs are given in the Appendix. Throughout the article, we use "increasing" and "decreasing" in nonstrict sense, that is, they represent "non-decreasing" and "non-increasing", respectively. In addition, for any real number $x$, we define $x^{+}=\max \{x, 0\}$ and $x^{-}=\max \{-x, 0\}$.

\section{THE MODEL}

Consider a capital-constrained firm that sells a product over a planning horizon of $N$ periods, indexed by $n=1, \ldots, N$. At the beginning of the planning horizon (i.e., period one), the firm has an initial capital $w_{1}$ and inventory stocking level $x_{1}$, with $w_{1}>0$ and $x_{1} \geq 0$. In each period $n$, the firm can replenish its inventory from a supplier with a unit cost $c$. The delivery leadtime is zero, implying that an order placed at the beginning of a period is delivered in the same period. The product has a fixed selling price $p$ and a random demand $D_{n}$ in period $n$. Following $\mathrm{Hu}$ et al. [11] and Li et al. [14], we assume $D_{1}, \ldots, D_{N}$ are independent and identically distributed nonnegative random variables, with $f(\cdot)$ and $F(\cdot)$ being their density and distribution functions, respectively. We also assume that any unsold product in a period is carried to the next period, any excess demand in a period is lost, and any unsold inventory at the end of the planning horizon is salvaged at a value $\gamma$ per unit with $\gamma \leq c$.

Besides using its own capital, the firm can borrow shortterm loans to finance its inventory in each period. The potential sources for such loans include the firm's shareholders, its supplier, third-party companies, or the banks. Note that if the short-term loans are from the firm's shareholders, then they can be regarded as (short-term) capital subscriptions; while if the loans are from the firm's supplier, then they can be regarded as trade credits. Let $\rho(z)$ denote the "total loan interest" in one period if the loan size is $z$, referred to as the "loan interest function." Then, $\rho^{\prime}(z)$ can be regarded as the "marginal interest function," and for convenience we shall refer to it as the "interest rate function." As the interest rate is positive and usually increases in the loan size, we assume that $\rho(z)$ is increasing and convex in $z$ with $\rho(0)=0$ and $\rho^{\prime}(0)>0$ being the minimum interest rate. In addition, the firm can deposit its extra capital into a savings account with a fixed interest rate $d$ per period. It is reasonable to assume $0 \leq d<\rho^{\prime}(0)$, as in practice the loan rate is always higher than the deposit rate. This assumption also ensures that the 
firm will never borrow a loan while deposit capital in the same period. We further assume $\rho^{\prime}(0)<(p-c) / c$, as otherwise the firm will never borrow and the problem then reduces to the model with a self-financing firm, which has been studied in Chao et al. [5]. Note that the loan interest function $\rho(z)$ described above includes many commonly used lending contracts as special cases. Two examples are: (1) a contract with a loan rate $b_{1}$ up to a loan size $K$ and a higher rate $b_{2}$ beyond $K$, specified by $\rho(z)=b_{1} z+\left(b_{2}-b_{1}\right)(z-K)^{+}$(where $\left.b_{2}>b_{1}\right)$; and (2) a contract with a borrowing cap $K$, given by any increasing convex function $\rho(z)$ but that is defined on the range $z \geq K$ by $\rho(z)=\rho(K)+(p-c)(z-K)^{+} / c$. In the latter case, the firm will never borrow more than $K$.

The sequence of events in each period $n$ is as follows: First, the firm reviews its inventory level $x_{n}$ and capital level $w_{n}$. Second, the firm decides on the order quantity for this period, denoted as $q_{n}$, pays the ordering cost $c q_{n}$ by its own capital and/or short-term loans, and deposits the extra capital (if any) to the savings account. Third, the order arrives and the inventory level is increased to $y_{n}$, with $y_{n}=x_{n}+q_{n}$. Fourth, the random demand $D_{n}$ is realized and the sales revenue $p \min \left\{y_{n}, D_{n}\right\}$ is collected. At the end of the period, the firm receives its deposited capital and interest from the savings account (if any), or pays the principal and interest of the short-term loan (if any). In case the firm does not have enough capital to pay the entire principal or interest of the loan, we assume that the firm pays all its capital and the remaining debt is carried to the next period as the firm's negative capital. Note that in this model, the inventory holding cost is implicitly included as the opportunity cost of capital and the shortage cost is implicitly included as the lost revenue. In addition, for simplicity we first assume in this section that there is no cost for physically holding the inventory; we will relax this assumption later in Section 4.1.

Let $\left(x_{n}, w_{n}\right)$ denote the state of the system in period $n$. Then, with the preceding description and assumptions of the model, the state transitions from period $n$ to period $n+1$ can be given as follows:

$$
\begin{aligned}
x_{n+1}= & \left(x_{n}+q_{n}-D_{n}\right)^{+} \\
w_{n+1}= & p \min \left\{x_{n}+q_{n}, D_{n}\right\}+(1+d)\left(w_{n}-c q_{n}\right)^{+} \\
& -\left(c q_{n}-w_{n}\right)^{+}-\rho\left(\left(c q_{n}-w_{n}\right)^{+}\right) .
\end{aligned}
$$

The transition of the inventory level follows directly from the lost-sales assumption; while the transition of the capital level consists of four terms. The first term on the right hand side of (2) is the sales revenue in period $n$, the second one is the capital received from the savings account, and the last two terms are the payments for the principal and interest of the shortterm loan, respectively. Note that under our assumptions, the firm deposits if and only if $w_{n} \geq c q_{n}$ and the deposit amount is $\left(w_{n}-c q_{n}\right)^{+}$; while it borrows if and only if $w_{n}<c q_{n}$ and the loan size is $\left(c q_{n}-w_{n}\right)^{+}$.
Note from (2) that even though the firm starts with a positive capital in period one (i.e., $w_{1}>0$ ), it may encounter a negative capital level in a future period (unless the firm never borrows). Thus, an important issue is whether the firm is allowed to operate with a negative capital level. Following $\mathrm{Hu}$ and Sobel [10] and Li et al. [14], we assume that the firm continues to operate with a negative capital level. However, in contrast to these two studies, we will consider the case where the firm does not have to pay a default penalty. As discussed earlier, to prevent the firm from overborrowing, the lender can choose an appropriate loan interest function $\rho(z)$, for example, by choosing $\rho(z)=\rho(K)+(p-c)(z-K) / c$ on $z \geq K$, then the firm will never borrow more than $K$. In addition, the lender can choose its protection level by varying the value $K$. In particular, if $K=0$, then the lender opts to not engage in any business with the firm. In practice, $\rho(z)$ is probably an outcome of a negotiation process between the firm and its lender, which balances the benefits and risks of both parties. We will relax this assumption later in Section 4.2 and study the case when the firm enters a reorganization bankruptcy when its capital level is negative.

Having described the state, actions, and state transitions, we now turn to the objective of the firm. Following the many studies in mathematical finance (e.g., Karatzas [12]; Pliska [16]), we assume that the firm's objective is to maximize its expected terminal wealth (capital) at the end of the planning horizon. That is, the firm's decision problem is: for given $w_{1}>0$ and $x_{1} \geq 0$,

$$
\max _{y_{1}, \ldots, y_{N}} \mathrm{E}\left[w_{N+1}+\gamma x_{N+1}\right]
$$

subject to state transitions (1) and (2), and $y_{n}=x_{n}+q_{n} \geq x_{n}$, for $n=1, \ldots, N$.

Denote by $V_{n}(x, w)$, the maximum expected terminal wealth, given that the inventory level is $x$ and the capital level is $w$ at the beginning of period $n$. Then, the following dynamic program can be formulated to analyze the firm's decision problem (3): for $1 \leq n \leq N$,

$$
\begin{aligned}
V_{n}(x, w)= & \max _{y \geq x} \mathrm{E}\left[V _ { n + 1 } \left(\left(y-D_{n}\right)^{+}, p \min \left\{y, D_{n}\right\}\right.\right. \\
& +\phi(w-c(y-x)))]
\end{aligned}
$$

where $\phi(s)=(1+d) s^{+}-s^{-}-\rho\left(s^{-}\right)$, and the boundary condition is

$$
V_{N+1}(x, w)=w+\gamma x
$$

Although the firm only needs to make an ordering decision in each period, there exist complex trade-offs in making this decision. Similar to other stochastic inventory control problems, the main trade-off is still between too many and too few. However, in contrast to those problems without financing considerations, the cost and benefit of ordering a certain 
quantity in our problem is much more complex to analyze, resulting from the nonlinear financing (revenue) function $\phi(\cdot)$ and multiperiod interactions. By analyzing the structural properties of the problem, we will characterize the firm's optimal policy in the next section.

\section{OPTIMAL POLICY}

We begin our analysis by examining the structural properties of problem (4). The following lemma provides some important properties that will help characterize the firm's optimal ordering policy.

LEMMA 1: For $n=1, \ldots, N$, the following results hold:

i. $V_{n}(x-z, w+p z)$ is strictly increasing in $w$ and $z$;

ii. $V_{n}(x, w)$ is concave in $(x, w)$.

Lemma 1(i) shows that the firm's expected terminal wealth will be increased if it has more initial capital, and the firm always prefers to sell inventory in exchange of capital. Both results are intuitive. In addition, the concavity of $V_{n}(x, w)$ indicates that the marginal value of additional inventory (resp., capital) decreases if the firm has more initial inventory (resp., capital). This result is also intuitive, as the probability of selling additional inventory (resp., using additional capital to purchase product) decreases when the firm has more initial inventory (resp., capital).

With the above properties of the value function, we proceed to study the firm's optimal inventory policy. We first provide a simple upper bound for the firm's optimal ordering quantity. To this end, we define a threshold capital level $\underline{w}$ as follows: if the convex function $(p-c) w / c+\rho(-w)$ is strictly increasing in $w$ on $w \leq 0$, then $\underline{w}=-\infty$; otherwise, $\underline{w}$ is defined as the largest minimizer of $(p-c) w / c+\rho(-w)$ over $w \leq 0$. Since $\rho(\cdot)$ is convex, $\underline{w}$ is uniquely defined with $\underline{w} \leq 0$.

PROPOSITION 1: For $n=1, \ldots, N$, given the state $(x, w)$ at the beginning of period $n$, the firm will not order more than $(w-\underline{w})^{+} / c$.

According to Proposition 1, if $w \leq \underline{w}$ in period $n$, then it is optimal for the firm not to order in period $n$ regardless of the starting inventory level in this period. The intuition behind this result is as follows. When $w \leq w$, the firm is in debt of $-w$ at the beginning of period $n$; thus it must resort to shortterm loan for ordering, with a marginal financing cost at least $1+\rho^{\prime}(-w)$ per unit of capital. On the other hand, the marginal revenue of ordering is at most $p / c$ per unit of capital, where $p / c$ is the price to cost ratio for the product. From the definition of $\underline{w}$, it is known that $1+\rho^{\prime}(-w)$ is greater than $p / c$ when $w \leq \underline{w}$. Thus, in this case it is not justifiable to borrow from the lender and order any quantity. Proposition 1 also shows that, if $w>\underline{w}$, then the firm's optimal ordering quantity has an upper bound $(w-\underline{w}) / c$, which is equivalent to requiring the firm's debt level after ordering to be no lower than $\underline{w}$. This result is consistent with the first result, as the firm has no incentive to order more product once its capital level drops to $\underline{w}$. Observe that $\underline{w}$ only depends on the loan interest function $\rho(\cdot)$ and the profit to cost ratio $(p-c) / c$. Thus, the lender of the short-term loan can indirectly control the firm's borrowing limit via the selection of $\rho(\cdot)$.

After obtaining the upper bound of the optimal ordering quantity, we proceed to characterize the firm's optimal inventory policy. It can be seen that the objective function on the right hand side of (4) depends on the state variables $x$ and $w$ only through $w+c x$. For convenience, for period $n$ with state $\left(x_{n}, w_{n}\right)$, we denote $R_{n}=w_{n}+c x_{n}$. Intuitively, $R_{n}$ can be interpreted as the "equity level" of the firm with on-hand inventory evaluated at the purchasing $\operatorname{cost} c$. Then, the optimality Eq. (4) can be rewritten, after introducing a new value function $\tilde{V}_{n}(x, R)$, as

$$
\tilde{V}_{n}(x, R):=V_{n}(x, w)=\max _{y \geq x} \pi_{n}(y, R),
$$

where

$$
\begin{aligned}
\pi_{n}(y, R)= & \mathrm{E}\left[\tilde { V } _ { n + 1 } \left(\left(y-D_{n}\right)^{+},\right.\right. \\
& \left.\left.(p-c) \min \left\{y, D_{n}\right\}+\phi(R-c y)+c y\right)\right] .
\end{aligned}
$$

For any $R$, let $y_{n}^{*}(R)$ be the maximizer of the optimization problem $\max _{y \geq 0} \pi_{n}(y, R)$. Following Lemma 1 , it is easy to show that $\pi_{n}(y, R)$ is concave in $(y, R)$; thus, $\pi_{n}(y, R)$ is increasing in $y$ when $y \leq y_{n}^{*}(R)$ and it is decreasing in $y$ when $y \geq y_{n}^{*}(R)$. Hence, the firm's optimal inventory policy can be characterized as follows.

THEOREM 1: For $n=1, \ldots, N$, given the state $(x, w)$ at the beginning of period $n$, an equity-level-dependent basestock inventory policy $y_{n}^{*}(R)$, where $R=w+c x$, is optimal. Specifically,

i. if $x<y_{n}^{*}(R)$, then it is optimal to replenish the inventory level to $y_{n}^{*}(R)$;

ii. if $x \geq y_{n}^{*}(R)$, then it is optimal not to order.

Theorem 1 shows that the firm's optimal order-up-to level depends on its equity level $R$ rather than the inventory level $x$ and the capital level $w$ individually. However, it does not characterize the properties of $y_{n}^{*}(R)$ as a function of $R$. For example, it does not specify the relationship between $y_{n}^{*}(R)$ and $R / c$. If $y_{n}^{*}(R) \leq R / c$, then the optimal ordering policy only uses the firm's own capital and does not borrow from the lender. In that case, we would have either $x \geq y_{n}^{*}(R)$ or 
$x<y_{n}^{*}(R)$, and only in the latter case ordering (using the firm's own capital) is necessary. In contrast, if $y_{n}^{*}(R)>R / c$, then the firm has to borrow from the lender to reach the optimal order-up-to level. In this case, if $x \geq y_{n}^{*}(R)$, then the firm has a negative capital level $w$ at the beginning of period $n$ and it is optimal not to order; while if $x<y_{n}^{*}(R)$, then the firm needs to borrow from the lender to place an order of quantity $y_{n}^{*}(R)-x$.

In what follows, we will focus on studying the properties of $y_{n}^{*}(R)$ for different values of $R$. First, the following result provides an upper bound for $y_{n}^{*}(R)$, which also shows that the optimal order-up-to level is equal to zero when the equity level $R$ is below $w$.

COROLLARY 1: For $n=1, \ldots, N, y_{n}^{*}(R) \leq(R-$ $\underline{w})^{+} / c$. In particular, $y_{n}^{*}(R)=0$ when $R \leq \underline{w}$.

Corollary 1 is a direct application of Theorem 1 and Proposition 1 . To see this, suppose that the state is $(x, R-c x)$ at the beginning of period $n$. By Theorem 1, the firm's optimal ordering quantity is $\left(y_{n}^{*}(R)-x\right)^{+}$. Since the firm's optimal order quantity at this state has an upper bound $(R-c x-\underline{w})^{+} / c$ by Proposition 1 , it follows that $\left(y_{n}^{*}(R)-\right.$ $x)^{+} \leq(R-c x-\underline{w})^{+} / c$. Then, the corollary directly follows by setting $x=0$.

We next study the properties of $y_{n}^{*}(R)$ when $R>\underline{w}$. We begin by defining two sequences of concave functions and two sequences of control parameters as follows: $G_{N+1}^{d}(y)=$ $(\gamma-c) y$, and for $n=1, \ldots, N$,

$$
\begin{aligned}
G_{n}^{d}(y)= & (1+d)^{N-n}\left((p-c) \mathrm{E}\left[\min \left\{y, D_{n}\right\}\right]-d c y\right) \\
& +\mathrm{E}\left[G_{n+1}^{d}\left(\max \left\{a_{n+1}^{d},\left(y-D_{n}\right)^{+}\right\}\right)\right], \\
G_{n}^{\mathrm{br}}(y)= & G_{n}^{d}(y)-(1+d)^{N-n}\left(\rho^{\prime}(0)-d\right) c y,
\end{aligned}
$$

where $a_{N+1}^{d}=0$, and for $n=1, \ldots, N, a_{n}^{d}$ and $a_{n}^{\text {br }}$ are maximizers of $G_{n}^{d}(y)$ and $G_{n}^{\mathrm{br}}(y)$ over $y \geq 0$, respectively. Note that $G_{n}^{d}(y)$ and $a_{n}^{d}$ are independent of the loan interest function $\rho(\cdot)$; while $G_{n}^{\text {br }}(y)$ and $a_{n}^{\text {br }}$ depend on $\rho(\cdot)$, but only through the minimum loan interest rate $\rho^{\prime}(0)$. As will be seen later, the two functions $G_{n}^{d}(y)$ and $G_{n}^{\mathrm{br}}(y)$ play determining roles in the strategy of the firm on the range of equity levels $R \geq \mathrm{ca}_{n}^{\mathrm{br}}$; more specifically, $\mathrm{ca}_{n}^{d}$ and $\mathrm{ca}_{n}^{\mathrm{br}}$ are the critical points of the equity levels for the firm to set its ordering strategy as if there were infinite capital and to use up all capital to replenish inventory but without borrowing, respectively. The following lemma presents some important properties on $a_{n}^{d}$ and $a_{n}^{\mathrm{br}}$.

LEMMA 2: The control parameters $a_{n}^{d}$ and $a_{n}^{\text {br }}, n=$ $1, \ldots, N$, satisfy

$$
\begin{aligned}
\text { i. } & F^{-1}\left(\frac{p-(1+d) c}{p-c}\right) \geq a_{1}^{d} \geq a_{2}^{d} \geq \cdots \geq a_{N}^{d}= \\
& F^{-1}\left(\frac{p-(1+d) c}{p-\gamma}\right) ; \\
\text { ii. } & F^{-1}\left(\frac{p-\left(1+\rho^{\prime}(0)\right) c}{p-c}\right) \geq a_{1}^{\text {br }} \geq a_{2}^{\text {br }} \geq \cdots \geq a_{N}^{\text {br }}= \\
& F^{-1}\left(\frac{p-\left(1+\rho^{\prime}(0)\right) c}{p-\gamma}\right) ;
\end{aligned}
$$

iii. for $n=1, \ldots, N, a_{n}^{\text {br }}$ is decreasing in $\rho^{\prime}(0)$, with $a_{n}^{\text {br }} \leq a_{n}^{d}$; in addition, for $n=1, \ldots, N-1$, if $a_{n+1}^{d} \leq F^{-1}\left(\frac{p-\left(1+\rho^{\prime}(0)\right) c}{p-c}\right)$, then $a_{n}^{\text {br }} \geq a_{n+1}^{d}$; otherwise, $a_{n}^{\mathrm{br}}=F^{-1}\left(\frac{p-\left(1+\rho^{\prime}(0)\right) c}{p-c}\right)$;

where $F^{-1}(\cdot)$ is the inverse function of the distribution function $F(\cdot)$.

According to Lemma $2, a_{1}^{d}, \ldots, a_{N}^{d}$ and $a_{1}^{\mathrm{br}}, \ldots, a_{N}^{\mathrm{br}}$ are two sequences of decreasing numbers, with $a_{n}^{d} \geq a_{n}^{\text {br }}>0$ for each $n$. With these numbers, the following theorem shows that, for each period $n, y_{n}^{*}(R)$ exhibits a very simple structure when $R \geq \mathrm{ca}_{n}^{\mathrm{br}}$.

THEOREM 2: For $n=1, \ldots, N$, the following results hold:

i. when $R \geq \mathrm{ca}_{n}^{d}, y_{n}^{*}(R)=a_{n}^{d}$; and when $\mathrm{ca}_{n}^{\mathrm{br}} \leq R \leq$ $\mathrm{ca}_{n}^{d}, y_{n}^{*}(R)=R / c$;

ii. when $R \geq \mathrm{ca}_{n}^{\mathrm{br}}$ and $R \geq c x$, the value function $\tilde{V}_{n}(x, R)$ can be decomposed as

$$
\tilde{V}_{n}(x, R)=G_{n}^{d}\left(\max \left\{a_{n}^{d}, x\right\}\right)+H_{n}(R),
$$

where

$$
H_{n}(R)=\left\{\begin{array}{cc}
\pi_{n}(R / c, R)-G_{n}^{d}\left(a_{n}^{d}\right), & \mathrm{ca}_{n}^{\mathrm{br}} \leq R \leq \mathrm{ca}_{n}^{d} ; \\
(1+d)^{N-n+1} R, & R \geq \mathrm{ca}_{n}^{d} .
\end{array}\right.
$$

Theorem 2 shows that the firm's optimal order-up-to level $y_{n}^{*}(R)$ is the constant $a_{n}^{d}$ when $R \geq \mathrm{ca}_{n}^{d}$. This is the case when the firm has a high-equity level $R$, resulting from either a high-inventory level $x$ or a high-capital level $w$. In this case, since $y_{n}^{*}(R) \leq R / c$, it is optimal for the firm to either not order (if $x \geq a_{n}^{d}$ ) or raise its inventory level to $a_{n}^{d}$ by using its own capital (if $x<a_{n}^{d}$ ). Since $a_{n}^{d}$ is independent of the firm's equity level $R$ and the loan interest function $\rho(\cdot)$, the firm having a high-equity level totally ignores its capital status and acts like a firm without the capital constraint in this period.

When $\mathrm{ca}_{n}^{\text {br }} \leq R \leq \mathrm{ca}_{n}^{d}$, Theorem 2 shows that the firm's optimal order-up-to level $y_{n}^{*}(R)$ is $R / c$. This is the case when the firm has a moderate-equity level, which is not high enough for the firm to ignore its capital status but is still sufficient to avoid the short-term loan. In this case, it is optimal for the firm to either not order (if $w \leq 0$ ) or raise its inventory 
level to $R / c$ by using up all its capital (if $w>0$ ). Thus, the firm with a moderate equity level does not need to resort to short-term financing and acts like a self-financing firm in this period. Note from Lemma 2 that the critical equity level $c a_{n}^{\text {br }}$ decreases in the minimum loan interest rate, $\rho^{\prime}(0)$. This is quite intuitive, as the firm is more reluctant to borrow if the introductory loan rate becomes higher.

The second part of Theorem 2 indicates that, if the firm has no debt and meanwhile owns a sufficient equity at the beginning of a period, then its optimal expected terminal wealth is independently determined by its inventory level and its equity level. This decomposition result eventually leads to the simple structure of the firm's optimal order-up-to level when its equity level is high or moderate. In contrast, when the firm's equity level is low, the decomposition result does not hold anymore; and as a result, as will be seen later, the firm's optimal order-up-to level becomes more complicated.

Having shown the structure of $y_{n}^{*}(R)$ for the ranges $R \leq \underline{w}$ and $R \geq \mathrm{ca}_{n}^{\mathrm{br}}$, we now study the remaining case when $\underline{w}<R<\mathrm{ca}_{n}^{\mathrm{br}}$. We start our analysis from the last period $N$, where the result is given in the following proposition.

PROPOSITION 2: For the last period $N$, when $\underline{w}<$ $R<\mathrm{ca}_{N}^{\text {br }}$, the firm's optimal order-up-to level $y_{N}^{*}(R)$ is an increasing function of $R$, given by

$$
\begin{aligned}
& y_{N}^{*}(R) \\
& \quad=\sup \left\{y \geq R^{+} / c \mid F(y) \leq \frac{p-c\left(1+\rho^{\prime}(c y-R)\right)}{p-\gamma}\right\} .
\end{aligned}
$$

From Proposition 2, when $\underline{w}<R<\mathrm{ca}_{N}^{\mathrm{br}}, y_{N}^{*}(R)$ is determined by the distribution function of the demand and the loan rate function. Given that the firm is solving a newsvendor-type problem in the last period, $y_{N}^{*}(R)$ could be interpreted as the critical fractile solution with a unit underage cost of $p-c\left(1+\rho^{\prime}(c y-R)\right)$ and a unit overage cost of $c\left(1+\rho^{\prime}(c y-R)\right)-\gamma$, where both costs depend on the loan size $c y-R$. As a special case of Proposition 2, when the interest function $\rho(\cdot)$ is piecewise linear, that is, for some sequences of increasing numbers $b_{i}$ and $K_{i}$ satisfying $d<b_{1}<\cdots<b_{m+1}=(p-c) / c$ and $0<K_{1}<\cdots<K_{m}$, $\rho(\cdot)$ is given by

$$
\rho(z)=b_{1} z+\sum_{i=1}^{m}\left(b_{i+1}-b_{i}\right)\left(z-K_{i}\right)^{+},
$$

we can compute $y_{N}^{*}(R)$ in a simple explicit form. In this case, the interest rate function $\rho^{\prime}(\cdot)$ is a step function and (9) can be greatly simplified. To present the result, let $a_{N}^{b_{i}}=$ $F^{-1}\left(\frac{p-c\left(1+b_{i}\right)}{p-\gamma}\right), i=1, \ldots, m$. Then, it can be shown that $0<a_{N}^{b_{m}}<\cdots<a_{N}^{b_{1}}$
COROLLARY 2: If the loan interest function is piecewise linear, then when $-K_{m}=\underline{w}<R<\mathrm{ca}_{N}^{\mathrm{br}}=\mathrm{ca}_{N}^{b_{1}}$, the optimal order-up-to level $y_{N}^{*}(R)$ is given by

$$
\begin{aligned}
& y_{N}^{*}(R) \\
& =\left\{\begin{array}{cc}
\left(R+K_{m}\right) / c, & -K_{m}<R<\mathrm{ca}_{N}^{b_{m}}-K_{m} ; \\
a_{N}^{b_{m}}, & \mathrm{ca}_{N}^{b_{m}}-K_{m} \leq R<\mathrm{ca}_{N}^{b_{m}}-K_{m-1} ; \\
\left(R+K_{m-1}\right) / c, & \mathrm{ca}_{N}^{b_{m}}-K_{m-1} \leq R<\mathrm{ca}_{N}^{b_{m-1}}-K_{m-1} ; \\
\vdots & \vdots \\
a_{N}^{b_{1}}, & \mathrm{ca}_{N}^{b_{1}}-K_{1} \leq R<\mathrm{ca}_{N}^{b_{1}} .
\end{array}\right.
\end{aligned}
$$

Therefore, when the loan interest function $\rho(\cdot)$ is a piecewise linear convex function with $m+1$ pieces, the firm's optimal order-up-to level $y_{N}^{*}(R)$ on $R \in\left(\underline{w}, \mathrm{ca}_{N}^{\mathrm{br}}\right)$ is completely characterized by $m$ constants and $m$ affine functions of $R$. The $m$ constants correspond to the ideal base-stock levels with the $m$ different loan rates $b_{1}, \ldots, b_{m}$; while the $m$ affine functions satisfy $c y_{N}^{*}(R)-R=K_{i}, i=1, \ldots, m$, and they are determined by the $m$ kinks of $\rho(z)$ when $z=K_{1}, \ldots, K_{m}$.

We now proceed to study, for a general period $n$, the structure of $y_{n}^{*}(R)$ on the range $\underline{w}<R<\mathrm{ca}_{n}^{\mathrm{br}}$. Given the result in Proposition 2 for the last period, it is conceivable that $y_{n}^{*}(R)$ does not have a simple structure when $\underline{w}<R<\mathrm{ca}_{n}^{\mathrm{br}}$. Then, what can be said about the optimal order-up-to level for this range? Toward this end, we first use the following numerical example to explore some insights.

EXAMPLE 1: Suppose the planning horizon $N=3$, the selling price of the product $p=1.3$, the unit cost $c=1$, the salvage value $\gamma=0.5$, and the demand in each period is uniformly distributed over the interval $[0,1]$. The deposit interest rate $d=0.05$, and the loan interest function $\rho(z)=0.1 z$. That is, the lender uses a flat loan rate of 0.1 and no borrowing limit. In this example, $\underline{w}=-\infty$, and the control parameters for period two are $a_{2}^{\text {br }}=0.5775$ and $a_{2}^{d}=0.6705$. The firm's optimal order-up-to level in period two, $y_{2}^{*}(R)$, as a function of its equity level $R$ is shown in Fig. 1 .

Figure 1 shows that $y_{2}^{*}(R)$ has a simple structure when $R \geq \mathrm{ca}_{2}^{\mathrm{br}}$, which is consistent with our analytical results in Theorem 2. When $R \leq \mathrm{ca}_{2}^{\text {br }}$, however, $y_{2}^{*}(R)$ is complicated and even not monotone in $R$. Recall that $y_{3}^{*}(R)$ is increasing in $R$ by Proposition 2. Thus, $y_{n}^{*}(R)$ is in general not monotone in $R$ except for the last period $N$. The implication of these results is that, while it is always optimal for the firm to order more in the last period when it has more capital, it can be optimal for the firm to order less when it has more capital and when there is more than one remaining period. Intuitively, this complex optimal ordering behavior of the firm is a result of two competing effects of having more capital. The positive effect is that, with more capital in hand the firm will need less short-term loan, thus it leads to lower financing cost to 


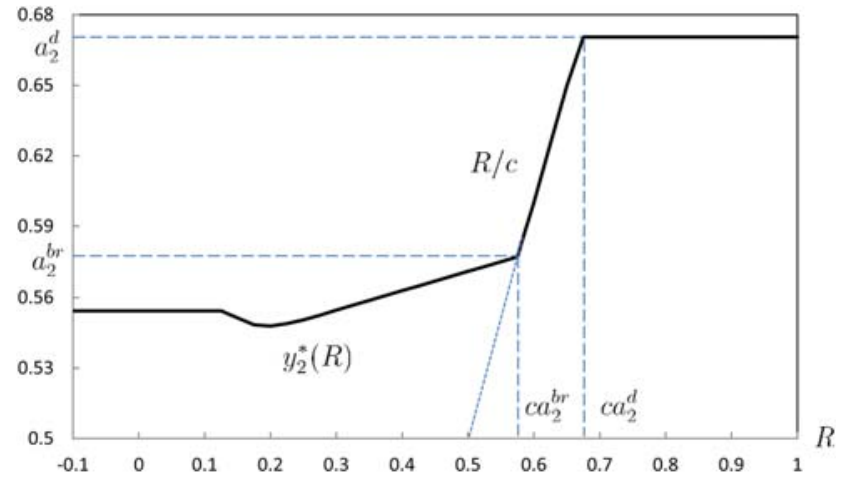

Figure 1. Optimal order-up-to level $y_{2}^{*}(R)$. [Color figure can be viewed in the online issues which is available at wileyonlinelibrary.com.]

achieve the same order-up-to level, which induces the firm to order more. In contrast, the negative effect of having more capital is that, the marginal value of revenue from sales would decrease due to the concavity of the value function, thus satisfying demand would become less attractive, which induces the firm to order less. Our results above show that the first effect always dominates the second in a single-period problem, leading to a monotone optimal order-up-to level with regard to the firm's capital. However, if there are multiple periods to go, then either effect can dominate the other one, resulting in the nonmonotone behavior as demonstrated in Figure 1.

Besides its complexity, Figure 1 also demonstrates two interesting properties of the optimal order-up-to level $y_{2}^{*}(R)$. First, $y_{2}^{*}(R) \geq R / c$ when $R \leq \mathrm{ca}_{2}^{\mathrm{br}}$; and second, $y_{2}^{*}(R)$ becomes flat when $R$ is sufficiently low. An interesting question is that, to what extent these two properties remain true for the general setting. In the rest of this section, we will address these two issues.

As discussed earlier in this section, the first property implies that when $R \leq c a_{2}^{\mathrm{br}}$, the firm has to borrow from the lender to reach the optimal order-up-to level $y_{2}^{*}(R)$ in period two. The following result summarizes our findings on the comparison between the optimal order-up-to level for period $n, y_{n}^{*}(R)$, and $R / c$.

PROPOSITION 3: For $n=N$ and $N-1, y_{n}^{*}(R) \geq R / c$ when $R \leq \mathrm{ca}_{n}^{\text {br }}$. Moreover, if the interest rate $d=0$, then the above result holds for $n=1, \ldots, N$.

Proposition 3 shows that the first property observed in the numerical example is always satisfied for the last two periods. In addition, when there is no savings account for deposit (equivalent to the case with $d=0$ ), this property is satisfied for an arbitrary period $n$. Unfortunately, for the case with a savings account of positive interest rate $d$, we are unable to obtain this property when there are three or more remaining periods. In that case, because of the complex multiperiod interactions, it is not clear which of the following options is more profitable: (1) use up all the firm's capital and even borrow some from the lender to build up inventory; (2) use part of the firm's capital to build up inventory while deposit the remaining capital to earn interest. Option (1) always dominates option (2) when the firm cannot earn deposit interest; however, there is no clear dominating relationship between these two options when there is a positive deposit rate and more than two periods remain.

We next discuss the second property exhibited in Figure 1, that is, the optimal order-up-to level $y_{n}^{*}(R)$ is a constant for small equity level $R$. From the result of Proposition 2, it can be seen that for an arbitrary increasing convex interest function $\rho(\cdot)$, this second property is not satisfied even for the last period. Hence, it probably results from our adoption of a linear loan interest function. Given that the linear loan interest function is the simplest loan scheme the lender could use, it is interesting to investigate whether the second property is generally true under this specific setting. Therefore, in the rest of this section, we will focus on the linear loan interest function: $\rho(z)=\rho^{\prime}(0) z=b z$, with $d<b<(p-c) / c$. Note by Theorem 2 that, $y_{n}^{*}(R)=\min \left\{a_{n}^{d}, R / c\right\}$ when $R \geq \mathrm{ca}_{n}^{\text {br }}$, $n=1, \ldots, N$. In the following, we show that for the linear loan interest function, the optimal order-up-to level $y_{n}^{*}(R)$ in period $n$ is always equal to a constant when the equity level $R$ is sufficiently low.

Before stating our main result, we need to introduce two more sequences of control parameters. First, denote $a_{N+1}^{b}=$ 0 , and for $n=1, \ldots, N$, denote $a_{n}^{b}$ as the maximizer of $G_{n}^{b}(y)$ over $y \geq 0$, where

$$
\begin{aligned}
G_{n}^{b}(y)= & (1+b)^{N-n}\left((p-c) \mathrm{E}\left[\min \left\{y, D_{n}\right\}\right]-b c y\right) \\
& +\mathrm{E}\left[G_{n+1}^{b}\left(\max \left\{a_{n+1}^{b},\left(y-D_{n}\right)^{+}\right\}\right)\right],
\end{aligned}
$$

and $G_{N+1}^{b}(y)=(\gamma-c) y$. Second, denote $a_{N+1}^{\mathrm{bl}}=\infty$, $a_{N}^{\mathrm{bl}}=a_{N}^{b}$, and for $n=1, \ldots, N-1$,

$$
a_{n}^{\mathrm{bl}}=\frac{\mathrm{ca}_{n+1}^{\mathrm{bl}}-(p-(1+b) c) a_{n}^{b}}{(1+b) c} .
$$

The following lemma establishes some important properties on these control parameters.

LEMMA 3: The control parameters $a_{n}^{b}$ and $a_{n}^{\text {bl }}, n=$ $1, \ldots, N$, satisfy

$$
\begin{aligned}
\text { i. } & F^{-1}\left(\frac{p-(1+b) c}{p-c}\right) \geq a_{1}^{b} \geq a_{2}^{b} \geq \cdots \geq a_{N}^{b}= \\
& F^{-1}\left(\frac{p-(1+b) c}{p-\gamma}\right) ; \\
\text { ii. }- & \frac{p-(1+b) c}{b c} F^{-1}\left(\frac{p-(1+b) c}{p-c}\right)<a_{1}^{\mathrm{bl}}<a_{2}^{\mathrm{bl}}<\cdots< \\
& a_{N}^{\mathrm{bl}}=F^{-1}\left(\frac{p-(1+b) c}{p-\gamma}\right) .
\end{aligned}
$$

Naval Research Logistics DOI 10.1002/nav 
Lemma 3 shows that, $a_{1}^{b}, \ldots, a_{N}^{b}$ is a sequence of decreasing positive numbers, while $a_{1}^{\mathrm{bl}}, \ldots, a_{N}^{\mathrm{bl}}$ is a sequence of increasing numbers. In addition, it follows from Lemmas 2 and 3 that $a_{N}^{b}=a_{N}^{\mathrm{bl}}=a_{N}^{\mathrm{br}}$, and $a_{n}^{\mathrm{bl}}<a_{n}^{\mathrm{br}}$ for $n=$ $1, \ldots, N-1$. With these control parameters, our main result is summarized in the following theorem.

THEOREM 3: If the loan interest function $\rho(z)=b z$, with $d<b<(p-c) / c$, then for $n=1, \ldots, N$,

i. when $R \leq \mathrm{ca}_{n}^{\mathrm{bl}}, y_{n}^{*}(R)=a_{n}^{b}$;

ii. when $R \leq \mathrm{ca}_{n}^{\mathrm{bl}}$ and $0 \leq x \leq \frac{\mathrm{ca}_{n+1}^{\mathrm{bl}}-(1+b) R}{p-(1+b) c}$, the value function $\tilde{V}_{n}(x, R)$ can be decomposed as

$$
\tilde{V}_{n}(x, R)=G_{n}^{b}\left(\max \left\{a_{n}^{b}, x\right\}\right)+(1+b)^{N-n+1} R .
$$

Theorem 3 shows that, under the linear loan interest function, the firm's optimal order-up-to level $y_{n}^{*}(R)$ in period $n$ is flat at $a_{n}^{b}$ when $R \leq \mathrm{ca}_{n}^{\mathrm{bl}}$. This confirms that the aforementioned second property is always true under a linear loan interest function. In this case, as $y_{n}^{*}(R) \geq a_{n}^{\mathrm{bl}} \geq R / c$, it is optimal for the firm to either not order (if $x \geq a_{n}^{b}$ ) or raise its inventory level to $a_{n}^{b}$ by borrowing from the lender (if $x<a_{n}^{b}$ ). Similar to the case with $R \geq \mathrm{ca}_{n}^{d}$, as $a_{n}^{b}$ does not depend on the firm's equity level $R$ and the deposit interest rate $d$, the firm with a low-equity level totally ignores its capital status and acts like a firm that is always in debt in this period. In addition, Theorem 3 (ii) provides a decomposition result similar to Theorem 2 (ii). Again, this result is crucial in establishing the constant optimal order-up-to level when the equity level is low.

With Theorems 2 and 3, the only interval of $R$ where $y_{n}^{*}(R)$ is not fully characterized under the linear loan interest function is $\left(\mathrm{ca}_{n}^{\mathrm{bl}}, \mathrm{ca}_{n}^{\mathrm{br}}\right)$. For the last period, since $a_{N}^{\mathrm{bl}}=a_{N}^{\mathrm{br}}$, $y_{N}^{*}(R)$ is fully characterized by these two theorems, with $y_{N}^{*}(R)=\max \left\{a_{N}^{b}, \min \left\{a_{N}^{d}, R / c\right\}\right\}$. When $n<N$, since $a_{n}^{\mathrm{bl}}$ is strictly increasing in $n$ while $a_{n}^{\text {br }}$ is decreasing in $n$ by Lemmas 2 and 3, this interval becomes strictly larger when $n$ decreases. Based on the upper bound of $a_{n}^{\text {br }}$ in Lemma 2 and the lower bound of $a_{n}^{\text {bl }}$ in Lemma 3, the width of the interval is upper bounded by $\frac{p-c}{b} F^{-1}\left(\frac{p-(1+b) c}{p-c}\right)$. For instance, in Example 1, $a_{n}^{\text {br }}$ has an upper bound $2 / 3, a_{n}^{\text {bl }}$ has a lower bound $-4 / 3$, and the width of the region $\left(\mathrm{ca}_{n}^{\mathrm{bl}}, \mathrm{ca}_{n}^{\mathrm{br}}\right)$ has an upper bound of 2. Furthermore, note that this upper bound is decreasing in $b$ and converges to 0 as $b$ tends to $(p-c) / c$. Therefore, under the linear loan interest function with a highinterest rate, the remaining interval where $y_{n}^{*}(R)$ is not fully characterized is quite narrow. On the other hand, as seen from the numerical example, in this narrow interval, the optimal policy cannot be expected to have a nice analytical structure. We suspect that in this interval, the optimal order-up-to level is decreasing first and then increasing (as observed in Figure 1), but we are unable to theoretically prove this.

Finally, we remark that, in the trivial case where the loan interest rate $b$ is equal to the deposit interest rate $d$, the firm's optimal inventory policy in each period is an equity-levelindependent base-stock policy and its value function can be completely decomposed. Specifically, for each period $n=$ $1, \ldots, N, y_{n}^{*}(R)=a_{n}^{d}$ and $\tilde{V}_{n}(x, R)=G_{n}^{d}\left(\max \left\{a_{n}^{d}, x\right\}\right)+$ $(1+d)^{N-n+1} R$ for any $x \geq 0$ and $-\infty<R<\infty$. This is because, when $d=b, \phi(s)=(1+d) s$ for all $s$, so the firm's marginal financing cost is a constant regardless of its capital level; thus there is no need to keep track of the capital level, and the resulting problem becomes a classical multiperiod newsvendor problem. This result can also be argued from a different perspective: if the loan rate is the same as the savings rate, then the firm can be considered as the owner of the bank that can freely borrow or deposit any amount of capital, hence the financing issue no longer exists in the firm's inventory management problem.

\section{EXTENSIONS}

In this section, we study three extensions of our base model, which are the model with physical inventory cost, the model with reorganization bankruptcy, and the model with fixed borrowing cost. For the first two extensions, we will show that most of our results and analysis continue to hold; while for the third extension, the problem is much more complicated and we characterize the optimal policy only for the last-period problem.

\subsection{Physical Holding Cost}

So far, we have assumed that there is no cost of physically holding the inventory; thus, the inventory holding cost only consists of the opportunity cost of capital. In this section, we relax this assumption and study a model with physical holding cost. Specifically, in addition to all the model assumptions in Section 2, we assume that the firm incurs a physical holding cost rate $h$ on unsold inventory at the end of each period. In the following, we show how our model and results are extended to this new model setting.

Because the physical holding cost only affects the firm's cash flow rather than its material flow, the state transition on the inventory in (1) remains the same, while the transition on the capital level in (2) needs to be modified as

$$
\begin{aligned}
w_{n+1}= & p \min \left\{x_{n}+q_{n}, D_{n}\right\}-h\left(x_{n}+q_{n}-D_{n}\right)^{+} \\
& +\phi\left(w_{n}-c q_{n}\right) .
\end{aligned}
$$

Accordingly, the dynamic program in (4) needs to be modified as: for $1 \leq n \leq N$, 


$$
\begin{aligned}
& V_{n}(x, w)=\max _{y \geq x} \mathrm{E}\left[V _ { n + 1 } \left(\left(y-D_{n}\right)^{+},\right.\right. \\
& \left.\left.p \min \left\{y, D_{n}\right\}-h\left(y-D_{n}\right)^{+}+\phi(w-c(y-x))\right)\right] .
\end{aligned}
$$

As in Section 3, we aim to characterize the firm's optimal ordering policy in each period. Following similar analysis to our base model, it is easy to show that Lemma 1, Proposition 1, Theorem 1, and Corollary 1 continue to hold under this new model setting. Therefore, an equity-level-dependent base-stock policy $y_{n}^{*}(R)$ remains optimal for period $n$, with $y_{n}^{*}(R)=0$ when $R \leq \underline{w}$. In addition, for the last period $N$, Theorem 2 remains true, and Proposition 2 and Corollary 2 also hold after replacing $\gamma$ by $\gamma-h$ (as after taking the physical holding cost into account, the "effective" salvage value for unsold product at the end of period $N$ becomes $\gamma-h$ ). For a general period $n$, after redefining some control parameters, Theorem 2 becomes partially true and Theorem 3 continues to hold. In the following, we present the results which are parallel to Theorems 2 and 3. Before that, we need to redefine the control parameters.

For $k=d, b$, denote $a_{N+1}^{k}=0$, and for $n=1, \ldots, N$, denote $a_{n}^{k}$ as the maximizer of $G_{n}^{k}(y)$ over $y \geq 0$, where

$$
\begin{aligned}
G_{n}^{k}(y)= & (1+k)^{N-n}\left((p-c+h) \mathrm{E}\left[\min \left\{y, D_{n}\right\}\right]\right. \\
& -(k c+h) y)+\mathrm{E}\left[G_{n+1}^{k}\left(\max \left\{a_{n+1}^{k},\left(y-D_{n}\right)^{+}\right\}\right)\right],
\end{aligned}
$$

and $G_{N+1}^{k}(y)=(\gamma-c) y$. In addition, for $n=1, \ldots, N, a_{n}^{\text {bl }}$ is defined in the same way as that in Section 3, except that in its definition $a_{n}^{b}$ should be replaced by what is defined in this section.

THEOREM 4: For $n=1, \ldots, N$, when $R \geq(c+$ $\left.\sum_{i=1}^{N-n}(1+d)^{-i} h\right) a_{n}^{d}, y_{n}^{*}(R)=a_{n}^{d}$. In addition, if $\rho(z)=b z$ with $d<b<(p-c) / c$, then for $n=1, \ldots, N, y_{n}^{*}(R)=a_{n}^{b}$ when $R \leq \mathrm{ca}_{n}^{\mathrm{bl}}$.

From Theorem 4, the firm's optimal order-up-to level $y_{n}^{*}(R)$ is still a constant when its equity level $R$ is above a certain level, and this level converges to that in Theorem 2 when $h$ reduces to 0 . In addition, for the special case with a linear loan interest function, $y_{n}^{*}(R)$ is also a constant when the equity level $R$ is below $\mathrm{ca}_{n}^{\mathrm{bl}}$, which is essentially the same as that in Theorem 3. For a general loan interest function, however, the characterization of $y_{n}^{*}(R)$ appears to be very complicated when $\underline{w}<R<\left(c+\sum_{i=1}^{N-n}(1+d)^{-i} h\right) a_{n}^{d}$, and it is difficult to obtain further results in this interval.

Furthermore, for the special case when $\rho(z)=d z$, the firm's optimal inventory policy in period $n$ is still an equitylevel-independent base-stock policy with the base-stock level $a_{n}^{d}$. This is because the firm's marginal financing cost remains a constant despite the physical holding cost. Therefore, there is still no need to keep track of the capital level, and the resulting problem is also a classical multiperiod newsvendor problem.

\subsection{Reorganization Bankruptcy}

In the preceding analysis, we have assumed that the firm does not go bankrupt when it ends up with a negative capital level in a period. In this section, we extend our base model to include the reorganization bankruptcy, or "Chapter 11" bankruptcy under the U.S. Bankruptcy Code. ${ }^{2}$ Under the reorganization bankruptcy, the firm continues to operate but incurs a default penalty cost if it has a negative capital level at the beginning of a period. Mathematically, following Li et al. [14], we assume that the firm incurs a default penalty $\eta_{n}\left(w_{n}\right)$ in state $\left(x_{n}, w_{n}\right)$ at the beginning of period $n$, where $\eta_{n}(w)$ is a convex decreasing function in $w$ with $\eta_{n}(w)=0$ when $w \geq 0, n=1, \ldots, N$. In addition, we assume that the firm's objective is to maximize its expected wealth minus default penalty at the end of the planning horizon, i.e., $\mathrm{E}\left[w_{N+1}+\gamma x_{N+1}-\eta_{N+1}\left(w_{N+1}+\gamma x_{N+1}\right)\right]$, where $\eta_{N+1}(w)$ is also a convex decreasing function in $w$ with $\eta_{N+1}(w)=0$ when $w \geq 0$. With other model assumptions of Section 2 remaining in place, we show in the following how our model and results are extended to this new model setting.

Since the default penalty only affects the firm's cash flow, the state transition on the inventory in (1) remains unchanged, while the capital level in (2) is modified to

$$
w_{n+1}=p \min \left\{x_{n}+q_{n}, D_{n}\right\}+\phi\left(w_{n}-\eta_{n}\left(w_{n}\right)-c q_{n}\right) .
$$

Accordingly, the dynamic program in (4) needs to be modified to: for $1 \leq n \leq N$,

$$
\begin{aligned}
V_{n}(x, w)= & \max _{y \geq x} \mathrm{E}\left[V _ { n + 1 } \left(\left(y-D_{n}\right)^{+},\right.\right. \\
& \left.\left.p \min \left\{y, D_{n}\right\}+\phi\left(w-\eta_{n}(w)-c(y-x)\right)\right)\right],
\end{aligned}
$$

and the boundary condition (5) is modified as $V_{N+1}(x, w)=$ $w+\gamma x-\eta_{N+1}(w+\gamma x)$.

We still aim to characterize the firm's optimal ordering policy in each period. With the reorganization bankruptcy, it is easy to show that Lemma 1 continues to hold. In addition, Proposition 1 and Theorem 1 are also true after $w$ being substituted with $w-\eta_{n}(w)$. This is because the effective capital level reduces from $w$ to $w-\eta_{n}(w)$ due to the default penalty $\eta_{n}(w)$ in period $n$. Thus, an equity-level-dependent base-stock policy $y_{n}^{*}(R)$ remains optimal for period $n$, where $R=w-\eta_{n}(w)+c x$. Consequently, the problem also reduces to the characterization of $y_{n}^{*}(R)$ for different values of $R$.

When $R \leq \underline{w}$, it is easy to show that Corollary 1 remains true thus $y_{n}^{*}(R)=0$. In addition, since $\eta_{n}(w)=0$ when $w \geq 0$ for $n=1, \ldots, N+1$, by following exactly the

\footnotetext{
${ }^{2}$ http://www.uscourts.gov/FederalCourts/Bankruptcy/ BankruptcyBasics/Process.aspx.
} 
same analysis as that for our base model, Theorem 2 continues to hold after introducing the default penalty. Hence, for each period $n, y_{n}^{*}(R)=\min \left\{a_{n}^{d}, R / c\right\}$ when $R \geq \mathrm{ca}_{n}^{\mathrm{br}}$. Thus, again the firm has an equity-level-independent constant optimal base-stock level as the equity level is high. This result implies that the default penalty does not affect the firm's optimal order-up-to level as long as it has a moderate or highequity level. For the remaining region when $\underline{w}<R<\mathrm{ca}_{n}^{\mathrm{br}}$, however, $y_{n}^{*}(R)$ depends on the default penalty functions and its characterization becomes much more complicated. Nevertheless, the comparison results between $y_{n}^{*}(R)$ and $R / c$ in Proposition 3 still hold. Thus, the insights generated from these results are still valid.

We remark that, for the last period $N$, the firm's decision problem when $R<\mathrm{ca}_{N}^{\mathrm{br}}$ is a special case of the risk-averse newsvendor problem studied by Eeckhoudt et al. [7]. Following their approach, it is easy to show that the firm's optimal order-up-to level $y_{N}^{*}(R)$ with the default penalty is lower than that without default penalty, that is, the expression given in (9). It means that the firm will order less after the reorganization bankruptcy is introduced, which is quite intuitive. In addition, unlike our base model and its extension with physical holding cost, the firm's problem cannot be reduced to a classical multiperiod newsvendor problem even if the loan interest function is $\rho(z)=d z$, that is, the borrowing rate is the same as the savings rate. This is because the firm incurs different default penalties at different capital levels; consequently, it must keep track of its capital level regardless of the structure of the loan interest function.

\subsection{Fixed Cost for Borrowing}

In this subsection, we study another extension of our model to include a fixed cost for borrowing. Specifically, we consider the case where there is an additional fixed cost $K$ for the firm when it borrows, which can be, for example, the processing cost of the loan.

When the fixed borrowing cost is in force, the state transition on the capital level in (2) is changed to

$$
\begin{aligned}
w_{n+1}= & p \min \left\{x_{n}+q_{n}, D_{n}\right\}+\phi\left(w_{n}-c q_{n}\right) \\
& -K \mathbf{1}\left\{c q_{n}>w_{n}^{+}\right\},
\end{aligned}
$$

where $\mathbf{1}\{\cdot\}$ is the indicator function, and $K \mathbf{1}\left\{c q_{n}>w_{n}^{+}\right\}$indicates that the fixed cost $K$ occurs when $c q_{n}>w_{n}^{+}$, or equivalently, the firm borrows capital in period $n$. Accordingly, the dynamic program in (4) is modified to: for $1 \leq n \leq N$,

$$
\begin{aligned}
V_{n}(x, w)= & \max _{y \geq x} \mathrm{E}\left[V _ { n + 1 } \left(\left(y-D_{n}\right)^{+}, p \min \left\{y, D_{n}\right\}\right.\right. \\
& \left.\left.+\phi(w-c(y-x))-K \mathbf{1}\left\{c(y-x)>w^{+}\right\}\right)\right],
\end{aligned}
$$

and the boundary condition remains $V_{N+1}(x, w)=w+\gamma x$.
With the introduction of the fixed borrowing cost, the value function $V_{n}(x, w)$ in (11) is not a concave function anymore, and nor can the objective function in (11) be written as a function of $y$ and $R=w+c x$. Consequently, the firm's optimal policy becomes much more complex and it is in general no longer an equity-level-dependent base-stock policy. To gain some insights on the optimal policy, we provide below its structure for the single-period model, that is, the last period $N$. Single-period models have many applications, hence the result below has independent interest, for example, inventory and financial decisions for the fashion industry.

PROPOSITION 4: For the last period $N$, when $w+c x \geq$ $\mathrm{ca}_{N}^{\mathrm{br}}$, it is optimal for the firm to raise its inventory level to as close to $a_{N}^{\text {br }}$ as possible by using its own capital only; while when $w+c x \leq \underline{w}$, it is optimal not to order. When $\underline{w}<w+c x<\mathrm{ca}_{N}^{\text {br }}$, then it is optimal to either order $w^{+} / c$ by using up its own capital or order up to $y_{N}^{*}(w+c x)$ [which is given by Eq. (9)], depending on which decision gives higher expected terminal wealth. In particular, when $\rho(z)=b z$ with $d \leq b<(p-c) / c$ and $w \geq 0$, then there exists a critical point $r^{*}$ such that it is optimal to order up to $a_{N}^{b}$ when $w+c x \leq r^{*}$; and it is optimal to order $w^{+} / c$ when $r^{*}<w+c x<\mathrm{ca}_{N}^{b}$.

Proposition 4 shows that when the fixed borrowing cost exists and the firm's equity level $R=w+c x$ falls into the region $\left(\underline{w}, \mathrm{ca}_{N}^{\mathrm{br}}\right)$, the firm makes a tradeoff between borrowing and not borrowing. When it is better not to borrow, then the firm's optimal decision is to use up its own capital to purchase inventory; otherwise, it is optimal to order up to an inventory level as if the fixed borrowing cost does not exist. In particular, when the loan interest rate is a constant and the firm has a nonnegative initial capital level, the proposition further shows that the optimal policy is an $(s, S)$-type policy, that is, it is optimal for the firm to borrow and raise the inventory level to a constant $a_{N}^{b}$ when its equity level is below a constant $r^{*}$. This result appears intuitive, because in the presence of the fixed cost, borrowing becomes unattractive if the amount to be borrowed is not sufficiently large.

\section{CONCLUSIONS}

In this article, we study a dynamic inventory control problem for a capital-constrained firm which can borrow shortterm loans to finance its inventory. By analyzing the structural properties of the firm's problem, we show that an equity-leveldependent base-stock inventory policy is optimal in each period, where the equity level is the sum of the firm's capital level and the value of its on-hand inventory evaluated at the purchasing cost. We further study the dependency of the firm's optimal base-stock level on its equity level, and show that optimal inventory policy is characterized by dividing the 
equity level into four intervals. We show that the optimal base-stock level is very simple when the equity level falls into three of the four intervals. More specifically, the optimal base-stock level is a constant when the equity level is in two of them, that is, when the equity level is either high or low; and it equals the amount of products the equity can purchase when it is in the third one. When the equity level is in the remaining interval, however, the optimal base-stock level is state-dependent and complicated. In this case, some partial characterization results are obtained. In particular, when the loan interest function is a linear function, we show that the interval where the optimal base-stock level is not fully characterized is quite narrow.

We also extend our model to the case with physical holding cost and the case with reorganization bankruptcy. In both cases, we show that most of the results for the base model continue to hold. In particular, an equity-level-dependent base-stock inventory policy is optimal for both cases. In fact, this result holds true under much more general settings, for example, when demands are independent but not identically distributed, and when the loan interest functions are timedependent. However, in these settings the characterization of the optimal base-stock level becomes more complicated due to the nonstationary data. Finally, a model with fixed borrowing cost is also considered and the optimal policy for the last period is analyzed.

Many interesting issues remain to be investigated. For example, the selling price is fixed in our model. In reality, the firm with abundant inventory but lack of capital tends to decrease the selling price so as to attract more demand. Thus, it is interesting to study the dynamic control problem of both inventory and price. Another interesting direction is to consider other types of bankruptcy (rather than the reorganization bankruptcy) when the firm's capital level drops below zero. For instance, under wipeout bankruptcy, or "Chapter 7" bankruptcy under the U.S. Bankruptcy Code, the firm with a negative capital level must halt operations and liquidate its assets. In this case, the firm's problem becomes an optimal stopping problem. Finally, the loan interest function in our model is independent of the firm's state (i.e., the inventory and capital levels). It is interesting to introduce such dependency as asset-based financing, which is typical in many applications. These are just a few possible future directions, and it appears that each of them will lead to a different optimal solution structure which is worthy of study.

\section{APPENDIX}

PROOF OF LEMMA 1: The proof is by induction. By the boundary condition (5), $V_{N+1}(x-z, w+p z)=(p-\gamma) z+\gamma x+w$. Since $p>\gamma$, the result obviously holds for $n=N+1$. Now assume inductively that it holds for $n+1$. In what follows, we prove it for $n$.
We first prove (i) for $n$. According to (4), we have

$$
\begin{aligned}
V_{n}(x-z, w+p z)= & \max _{y \geq x-z} \mathrm{E}\left[V _ { n + 1 } \left(\left(y-D_{n}\right)^{+},\right.\right. \\
& \left.\left.p \min \left\{y, D_{n}\right\}+\phi((p-c) z+w-c(y-x))\right)\right] .
\end{aligned}
$$

Since $\phi(s)=(1+d) s^{+}-\left(s^{-}+\rho\left(s^{-}\right)\right)$is strictly increasing in $s$ and $p>c$, $\phi((p-c) z+w-c(y-x))$ is strictly increasing in $w$ and $z$. In addition, since $V_{n+1}(x, w)$ is strictly increasing in $w$ according to the inductive assumption and the decision constraint $y \geq x-z$ is less restrictive when $z$ becomes larger, it follows that $V_{n}(x-z, w+p z)$ is strictly increasing in $w$ and $z$.

We next prove (ii) for $n$. For convenience, define $f_{n}(y, s, D)=$ $V_{n+1}\left((y-D)^{+}, p \min \{y, D\}+\phi(s)\right)$. Then, the optimality equation (4) can be rewritten as

$$
V_{n}(x, w)=\max _{y \geq x} \mathrm{E}\left[f_{n}\left(y, w-c(y-x), D_{n}\right)\right] .
$$

To prove the concavity of $V_{n}(x, w)$, we first prove that $f_{n}(y, s, D)$ is concave in $(y, s)$ for any given $D$. That is, for any $\left(y_{1}, s_{1}\right),\left(y_{2}, s_{2}\right)$ and $\lambda$, with $0 \leq \lambda \leq 1$, we need to show that

$$
\begin{aligned}
\lambda f_{n}\left(y_{1}, s_{1}, D\right)+(1-\lambda) f_{n}\left(y_{2},\right. & \left.s_{2}, D\right) \\
& \leq f_{n}\left(\lambda y_{1}+(1-\lambda) y_{2}, \lambda s_{1}+(1-\lambda) s_{2}, D\right) .
\end{aligned}
$$

Notice that for given $D$, both $\min \{y, D\}$ and $\phi(s)$ are concave functions. Then,

$$
\begin{aligned}
\lambda \min \left\{y_{1}, D\right\}+(1-\lambda) \min \left\{y_{2}, D\right\} & \leq \min \left\{\lambda y_{1}+(1-\lambda) y_{2}, D\right\} ; \\
\lambda \phi\left(s_{1}\right)+(1-\lambda) \phi\left(s_{2}\right) & \leq \phi\left(\lambda s_{1}+(1-\lambda) s_{2}\right) .
\end{aligned}
$$

Also notice that $(y-D)^{+}=y-\min \{y, D\}$. Then, it follows that

$$
\begin{aligned}
& \lambda f_{n}\left(y_{1}, s_{1}, D\right)+(1-\lambda) f_{n}\left(y_{2}, s_{2}, D\right) \\
&= \lambda V_{n+1}\left(\left(y_{1}-D\right)^{+}, p \min \left\{y_{1}, D\right\}+\phi\left(s_{1}\right)\right) \\
&+(1-\lambda) V_{n+1}\left(\left(y_{2}-D\right)^{+}, p \min \left\{y_{2}, D\right\}+\phi\left(s_{2}\right)\right) \\
& \leq V_{n+1}\left(\lambda\left(y_{1}-D\right)^{+}+(1-\lambda)\left(y_{2}-D\right)^{+}, \lambda\left(p \min \left\{y_{1}, D\right\}+\phi\left(s_{1}\right)\right)\right. \\
&\left.+(1-\lambda)\left(p \min \left\{y_{2}, D\right\}+\phi\left(s_{2}\right)\right)\right) \\
&= V_{n+1}\left(\left(\lambda y_{1}+(1-\lambda) y_{2}\right)-\left(\lambda \min \left\{y_{1}, D_{1}\right\}+(1-\lambda) \min \left\{y_{2}, D_{2}\right\}\right),\right. \\
&\left.\left(\lambda \phi\left(s_{1}\right)+(1-\lambda) \phi\left(s_{2}\right)\right)+p\left(\lambda \min \left\{y_{1}, D\right\}+(1-\lambda) \min \left\{y_{2}, D\right\}\right)\right) \\
& \leq V_{n+1}\left(\left(\lambda y_{1}+(1-\lambda) y_{2}-D\right)^{+}, p \min \left\{\lambda y_{1}+(1-\lambda) y_{2}, D\right\}\right. \\
&\left.+\phi\left(\lambda s_{1}+(1-\lambda) s_{2}\right)\right) \\
&= f_{n}\left(\lambda y_{1}+(1-\lambda) y_{2}, \lambda s_{1}+(1-\lambda) s_{2}, D\right),
\end{aligned}
$$

where the first inequality is from the inductive assumption that $V_{n+1}(x, w)$ is concave in $(x, w)$, and the second one is from the inductive assumption that $V_{n+1}(x-z, w+p z)$ is increasing in $w$ and $z$. Thus, $f_{n}(y, s, D)$ is concave in $(y, s)$.

Since $f_{n}(y, s, D)$ is concave in $(y, s)$, it follows that $f_{n}(y, w-c(y-x), D)$ is concave in $(y, x, w)$; and moreover, $\mathrm{E}\left[f_{n}\left(y, w-c(y-x), D_{n}\right)\right]$ is concave in $(y, x, w)$. Since the constraint $y \geq x$ in (12) is a convex set, by applying Proposition B-4 of Heyman and Sobel [8], we obtain that $V_{n}(x, w)$ is concave in $(x, w)$. Thus, the lemma holds for $n$. The proof is complete.

PROOF OF PROPOSITION 1: The proposition is trivial when $\underline{w}=-\infty$. Thus, we only prove the case when $-\infty<\underline{w} \leq 0$. By the optimality equation (4), it suffices to prove that, for any given $D$,

$$
\begin{aligned}
& \quad V_{n+1}\left((y-D)^{+}, p \min \{y, D\}+\phi(w-c(y-x))\right) \\
& \quad=V_{n+1}\left((y-D)^{+},-p(y-D)^{+}+p y+\phi(w-c(y-x))\right)
\end{aligned}
$$


is decreasing in $y$ when $y \geq x+(w-\underline{w})^{+} / c$. Notice from Lemma 1 that $V_{n+1}(x-z, w+p z)$ is increasing in $w$ and $z$. Thus, to obtain the desired result, it suffices to prove $p y+\phi(w-c(y-x))$ is decreasing in $y$ when $y \geq x+(w-\underline{w})^{+} / c$.

Now suppose $y \geq x+(w-\underline{w})^{+} / c$. In this case, $w-c(y-x) \leq$ $\min \{w, \underline{w}\} \leq \underline{w} \leq 0$. From the definition of $\phi(\cdot)$, we have

$$
\begin{gathered}
\quad p y+\phi(w-c(y-x))=(p-c) y+w+c x-\rho(-(w-c(y-x)) \\
=-[(p-c)(w-c(y-x)) / c+\rho(-(w-c(y-x))]+p x+p w / c .
\end{gathered}
$$

By the definition of $\underline{w}$ and the convexity of $\rho(\cdot),(p-c) s / c-\rho(-s)$ is decreasing in $s$ when $s \leq \underline{w}$. Hence, it follows that $p y+\phi(w-c(y-x))$ is decreasing in $y$ when $y \geq x+(w-\underline{w})^{+} / c$. The proof is complete.

PROOF OF LEMMA 2: The proof of (i) is exactly the same as that of Lemma 4 in Chao et al. [5]; thus it is omitted for brevity. In what follows, for convenience, we first prove (iii) and then prove (ii).

We first prove (iii). For $n=1, \ldots, N$, from (8), we have

$$
G_{n}^{d}(y)-G_{n}^{\mathrm{br}}(y)=c(1+d)^{N-n}\left(\rho^{\prime}(0)-d\right) y .
$$

Since $a_{n}^{d}$ and $a_{n}^{\mathrm{br}}$ are maximizers of $G_{n}^{d}(y)$ and $G_{n}^{\mathrm{br}}(y)$, respectively, it follows that

$$
\begin{aligned}
G_{n}^{d}\left(a_{n}^{d}\right)+G_{n}^{\mathrm{br}}\left(a_{n}^{\mathrm{br}}\right)-G_{n}^{\mathrm{br}}\left(a_{n}^{d}\right) & -G_{n}^{d}\left(a_{n}^{\mathrm{br}}\right) \\
& =c(1+d)^{N-n}\left(\rho^{\prime}(0)-d\right)\left(a_{n}^{d}-a_{n}^{\mathrm{br}}\right) \geq 0 .
\end{aligned}
$$

This implies $a_{n}^{d} \geq a_{n}^{\text {br }}$. In addition, according to (8), when $a_{n+1}^{d} \leq$ $F^{-1}\left(\frac{p-\left(1+\rho^{\prime}(0)\right) c}{p-c}\right)$, we have

$$
\left(G_{n}^{\mathrm{br}}\right)^{\prime}\left(a_{n+1}^{d}\right)=(1+d)^{N-n}\left((p-c) \bar{F}\left(a_{n+1}^{d}\right)-\rho^{\prime}(0) c\right) \geq 0 .
$$

Thus, it follows from the concavity of $G_{n}^{\mathrm{br}}(y)$ that $a_{n+1}^{d} \leq a_{n}^{\mathrm{br}}$. If $a_{n+1}^{d}>$ $F^{-1}\left(\frac{p-(1+b) c}{p-c}\right)$, then

$$
\left(G_{n}^{\mathrm{br}}\right)^{\prime}\left(F^{-1}\left(\frac{p-\left(1+\rho^{\prime}(0)\right) c}{p-c}\right)\right)=0 .
$$

This proves $a_{n}^{\mathrm{br}}=F^{-1}\left(\frac{p-\left(1+\rho^{\prime}(0)\right) c}{p-c}\right)$. Thus, (iii) is satisfied.

We now prove (ii). First, since $a_{N+1}^{d}=0$ and $G_{N+1}^{d}(y)=(\gamma-c) y$, it can be easily calculated from (8) that $a_{N}^{b}=F^{-1}\left(\frac{p-\left(1+\rho^{\prime}(0)\right) c}{p-\gamma}\right)$. Second, since $\mathrm{E}\left[G_{n+1}^{d}\left(\max \left\{a_{n+1}^{d},\left(y-D_{n}\right)^{+}\right\}\right)\right]$is decreasing in $y$, it can be easily seen from (7) and (8) that $a_{n}^{\text {br }} \leq F^{-1}\left(\frac{p-\left(1+\rho^{\prime}(0)\right) c}{p-c}\right)$. Finally, for $n=1, \ldots, N-1$, the inequality $a_{n}^{\mathrm{br}} \geq a_{n+1}^{\mathrm{br}}$ directly follows from part (iii) of the lemma. Thus, (ii) holds. The proof is complete.

PROOF OF THEOREM 2: We prove the theorem together with the additional result (iii) $H_{n}^{\prime}(R) \geq(1+d) H_{n+1}^{\prime}(R)$ on $R \geq \mathrm{ca}_{n}^{\mathrm{br}}$ by induction on $n$. The proof proceeds as follows. First, we prove that (i) holds for $N$, (ii) holds for $N$ and $N+1$, and (iii) holds for $N$. Then, by inductively assuming that (i) and (iii) hold for $n+1$ and (ii) holds for $n+1$ and $n+2$, we prove that (i), (ii) and (iii) hold for $n$.

We first prove that (i) and (iii) hold for $N$ and (ii) holds for $N$ and $N+1$. By (5) and $G_{N+1}^{d}(y)=(\gamma-c) y$, we have $a_{N+1}^{d}=0$. Thus, it follows that, when $R \geq 0$,

$$
\begin{aligned}
\tilde{V}_{N+1}(x, R) & =V_{N+1}(x, R-c x)=R+(\gamma-c) x \\
& =G_{N+1}^{d}\left(\max \left\{a_{N+1}^{d}, x\right\}\right)+H_{N+1}(R) .
\end{aligned}
$$

This shows that (ii) holds for $N+1$. When $n=N$, since $\tilde{V}_{N+1}(x, R)=$ $R+(\gamma-c) x$, it follows from (6), (7), and (8) that

$$
\begin{aligned}
\pi_{N}(y, R)= & (\gamma-c) \mathrm{E}\left[\left(y-D_{N}\right)^{+}\right] \\
& +(p-c) \mathrm{E}\left[\min \left\{y, D_{N}\right\}\right]+\phi(R-c y)+c y \\
= & \left\{\begin{array}{cc}
(1+d) R+G_{N}^{d}(y), & 0 \leq y \leq R / c ; \\
\left(1+\rho^{\prime}(0)\right) R+G_{N}^{\mathrm{br}}(y) & \\
-\left(\rho(c y-R)-\rho^{\prime}(0)(c y-R)\right), & y \geq R / c .
\end{array}\right.
\end{aligned}
$$

Note that $\pi_{N}(y, R)$ is concave in $y$ and $\rho(c y-R)-\rho^{\prime}(0)(c y-R)$ is increasing in $y$ on $y \geq R / c$. Then, when $R \geq \mathrm{ca}_{n}^{d}, y_{N}^{*}(R)=$ $\arg \max _{y \geq 0} \pi_{N}(y, R)=a_{N}^{d}$. When ca br $\leq R \leq \mathrm{ca}_{N}^{d}$, since $\pi_{N}(y, R)$ is increasing in $y$ on $0 \leq y \leq R / c$ and it is decreasing in $y$ on $y \geq R / c$, it follows that $y_{N}^{*}(R)=R / c$. Thus, (i) holds when $n=N$. In addition, when $R \geq c x$, we have

$$
\begin{aligned}
\tilde{\pi}_{N}(x, R) & =\pi_{N}\left(\max \left\{y_{N}^{*}(R), x\right\}, R\right) \\
& = \begin{cases}(1+d) R+G_{N}^{d}\left(\max \left\{a_{n}^{d}, x\right\}\right), & R \geq \mathrm{ca}_{n}^{d} ; \\
\pi_{N}(R / c, R), & \mathrm{ca}_{N}^{\mathrm{br}} \leq R \leq \mathrm{ca}_{N}^{d} .\end{cases}
\end{aligned}
$$

Thus, (ii) also holds when $n=N$. Finally, for any $R \geq \mathrm{ca}_{N}^{\mathrm{br}}$, since $H_{N}(R)$ is a concave function, we have $H_{N}^{\prime}(R) \geq 1+d=(1+d) H_{N+1}^{\prime}(R)$. Thus, (iii) also holds for $N$.

Now assume inductively that (i) and (iii) hold for $n+1$ and (ii) holds for $n+1$ and $n+2$. In what follows, we prove that (i), (ii) and (iii) hold for $n$, which then completes the proof.

First, we prove (i) holds for $n$. Suppose $R \geq \mathrm{ca}_{n+1}^{d}$ and $y \leq R / c$. Then, $R \geq \mathrm{ca}_{n+1}^{\mathrm{br}}$ by Lemma 2. By the inductive assumption on (ii) for $n+1$, we have

$$
\begin{aligned}
\pi_{n}(y, R) & =\mathrm{E}\left[\tilde{V}_{n+1}\left(\left(y-D_{n}\right)^{+},(p-c) \min \left\{y, D_{n}\right\}+\phi(R-c y)+c y\right)\right] \\
& =\mathrm{E}\left[\tilde{V}_{n+1}\left(\left(y-D_{n}\right)^{+},(p-c) \min \left\{y, D_{n}\right\}+(1+d) R-d c y\right)\right] \\
& =(1+d)^{N-n+1} R+G_{n}^{d}(y) .
\end{aligned}
$$

When $R>\mathrm{ca}_{n}^{d}$, since $a_{n}^{d} \geq a_{n+1}^{d}$, it follows from (13) that $\pi_{n}(y, R)$ is increasing in $y$ when $y \leq a_{n}^{d}$, and it is decreasing in $y$ when $a_{n}^{d} \leq y \leq R / c$. Note that $\pi_{n}(y, R)$ is concave in $y$ when $y \geq 0$. Thus, $y_{n}^{*}(R)=a_{n}^{d}$ when $R>\mathrm{ca}_{n}^{d}$.

Now suppose $\mathrm{ca}_{n}^{\mathrm{br}} \leq R \leq \mathrm{ca}_{n}^{d}$; and we shall prove that $y_{n}^{*}(R)=R / c$ in this case. By the definition of $y_{n}^{*}(R)$, it suffices to prove that $\pi_{n}(y, R)$ is increasing in $y$ when $y \leq R / c$, and $\pi_{n}(R / c, R)=\max _{y \geq R / c} \pi_{n}(y, R)$. In what follows, we prove these two results sequentially.

We first prove $\pi_{n}(y, R)$ is increasing in $y$ on $y \leq R / c$. When $\mathrm{ca}_{n+1}^{d} \leq$ $R \leq \mathrm{ca}_{n}^{d}, \pi_{n}(y, R)$ is given by (13) and the desired result is clearly true. Now suppose $\mathrm{ca}_{n}^{\mathrm{br}} \leq R \leq \mathrm{ca}_{n+1}^{d}$, and we prove that the desired result is also true in this case. Since $\pi_{n}(y, R)$ is concave in $y$, we only need to prove that $\left.\frac{\partial \pi_{n}(y, R)}{\partial y}\right|_{y=R / c} \geq 0$.

When $y \leq R / c$, by the inductive assumption on (ii) for $n+1$, we have

$$
\begin{aligned}
\pi_{n}(y, R)= & G_{n+1}^{d}\left(a_{n+1}^{d}\right) \\
& +\mathrm{E}\left[H_{n+1}\left((p-c) \min \left\{y, D_{n}\right\}+(1+d) R-d c y\right)\right] .
\end{aligned}
$$

Taking the partial derivative with respect to $y$ and letting $y=R / c$, we obtain

$$
\begin{aligned}
\frac{\partial \pi_{n}(R / c, R)}{\partial y} \\
=\mathrm{E}\left[\left((p-c) 1_{\left\{R / c \leq D_{n}\right\}}-d c\right) H_{n+1}^{\prime}\left((p-c) \min \left\{R / c, D_{n}\right\}+R\right)\right] \\
=(p-(1+d) c) \bar{F}(R / c) H_{n+1}^{\prime}(p R / c) \\
\quad-d c \mathrm{E}\left[1_{\left\{R / c \geq D_{n}\right\}} H_{n+1}^{\prime}\left((p-c) D_{n}+R\right)\right]
\end{aligned}
$$

Naval Research Logistics DOI 10.1002/nav 


$$
\begin{aligned}
& \geq(p-(1+d) c) \bar{F}(R / c) H_{n+1}^{\prime}(p R / c)-d c F(R / c) H_{n+1}^{\prime}(R) \\
& \geq\left((1+d)(p-c) \bar{F}(R / c) H_{n+2}^{\prime}(p R / c)-d c H_{n+1}^{\prime}(R)\right) F(R / c),
\end{aligned}
$$

where $1_{\{A\}}$ is the indicator function; the first inequality is from the concavity of $H_{n+1}(\cdot)$; and the second one is from the inductive assumption on (iii), $H_{n+2}(R)$ is increasing in $R$, and $R / c \leq a_{n+1}^{d} \leq F^{-1}\left(\frac{p-(1+d) c}{p-c}\right)$.

By (15), to prove $\frac{\partial \pi_{n}(R / c, R)}{\partial y} \geq 0$, it suffices to show that

$$
(1+d)(p-c) \bar{F}(R / c) H_{n+2}^{\prime}(p R / c) \geq d c H_{n+1}^{\prime}(R) .
$$

Using the inductive assumptions on (ii) for $n+1$ and $n+2$, we obtain

$$
\begin{aligned}
& \frac{\partial \pi_{n+1}(R / c, R)}{\partial y} \\
& \quad \leq \mathrm{E}\left[\left((p-c) 1_{\left\{R / c \leq D_{n+1}\right\}}-d c\right) H_{n+2}^{\prime}\left((p-c) \min \left\{R / c, D_{n+1}\right\}+R\right)\right],
\end{aligned}
$$

and

$$
\begin{aligned}
& H_{n+1}^{\prime}(R)=\frac{\mathbf{d} \pi_{n+1}(R / c, R)}{\mathbf{d} R} \\
& \quad \leq \mathrm{E}\left[\left(\frac{p-c}{c} 1_{\left\{R / c \leq D_{n+1}\right\}}+1\right) H_{n+2}^{\prime}\left((p-c) \min \left\{R / c, D_{n+1}\right\}+R\right)\right] .
\end{aligned}
$$

Combining the above two inequalities, we obtain

$$
\frac{\partial \pi_{n+1}(R / c, R)}{\partial y}+c d H_{n+1}^{\prime}(R) \leq(1+d)(p-c) \bar{F}(R / c) H_{n+2}^{\prime}(p R / c) .
$$

Since $\mathrm{ca}_{n}^{\mathrm{br}} \leq R \leq \mathrm{ca}_{n+1}^{d}$ and by the inductive assumption on (i), we have $\frac{\partial \pi_{n+1}(R / c, R)}{\partial y} \geq 0$. Thus, (16) holds. Therefore, when $\mathrm{ca}_{n}^{\mathrm{br}} \leq R \leq \mathrm{ca}_{n+1}^{d}$, $\pi_{n}(y, R)$ is increasing in $y$ on $y \leq R / c$.

We next prove $\pi_{n}(R / c, R)=\max _{y \geq R / c} \pi_{n}(y, R)$ when $\mathrm{ca}_{n}^{\mathrm{br}} \leq R \leq \mathrm{ca}_{n}^{d}$. For $y \geq 0$, we define

$\pi_{n}^{b}(y, R)=\mathrm{E}\left[\tilde{V}_{n+1}\left(\left(y-D_{n}\right)^{+},(p-c) \min \left\{y, D_{n}\right\}+R-\rho^{\prime}(0)(c y-R)\right)\right]$.

Then, by Lemma 1 , it is easy to verify that $\pi_{n}^{b}(y, R)$ is concave in $y$. When $y \geq R / c$, it follows from (6) that

$\pi_{n}(y, R)=\mathrm{E}\left[\tilde{V}_{n+1}\left(\left(y-D_{n}\right)^{+},(p-c) \min \left\{y, D_{n}\right\}+R-\rho(c y-R)\right)\right]$.

Since $\rho(\cdot)$ is a convex function, $\rho(c y-R) \geq \rho^{\prime}(0)(c y-R)$ when $y \geq R / c$. Then, by Lemma 1 (i), it follows that $\pi_{n}(y, R) \leq \pi_{n}^{b}(y, R)$ when $y \geq R / c$ and $\pi_{n}(R / c, R)=\pi_{n}^{b}(R / c, R)$. Therefore, to prove the desired result, it suffices to prove that $\pi_{n}^{b}(y, R)$ is decreasing in $y$ when $y \geq R / c-\epsilon$, with $\epsilon$ being a sufficiently small positive number, or equivalently, $\frac{\partial \pi_{n}^{b}(R / c, R)}{\partial y} \leq 0$. In what follows, we divide the analysis into two cases.

CASE 1: $R \geq c \max \left\{a_{n+1}^{d}, a_{n}^{\text {br }}\right\}$. In this case, when $y \leq R / c$, by the inductive assumption on (ii),

$$
\begin{aligned}
\pi_{n}^{b}(y, R)= & \mathrm{E}\left[\tilde { V } _ { n + 1 } \left(\left(y-D_{n}\right)^{+},(p-c) \min \left\{y, D_{n}\right\}+R\right.\right. \\
& \left.\left.+\rho^{\prime}(0)(R-c y)\right)\right] \\
= & (1+d)^{N-n}\left(1+\rho^{\prime}(0)\right) R+G_{n}^{\mathrm{br}}(y) .
\end{aligned}
$$

Since $a_{n}^{\mathrm{br}}=\arg \max _{y \geq 0} G_{n}^{\mathrm{br}}(y)$, it is obviously that $\frac{\partial \pi_{n}^{b}(R / c, R)}{\partial y} \leq 0$.
CASE 2: $\mathrm{ca}_{n}^{\mathrm{br}} \leq R<\mathrm{ca}_{n+1}^{d}$. In this case, by Lemma 2, $a_{n}^{\text {br }}=$ $F^{-1}\left(\frac{p-\left(1+\rho^{\prime}(0)\right) c}{p-c}\right)$. When $y \leq R / c$, by the inductive assumption on (ii),

$$
\begin{aligned}
& \pi_{n}^{b}(y, R)=\mathrm{E}\left[\tilde { V } _ { n + 1 } \left(\left(y-D_{n}\right)^{+},(p-c) \min \left\{y, D_{n}\right\}+R\right.\right. \\
& \left.\left.\quad+\rho^{\prime}(0)(R-c y)\right)\right] \\
& =G_{n+1}^{d}\left(a_{n+1}^{d}\right)+\mathrm{E}\left[H_{n+1}\left((p-c) \min \left\{y, D_{n}\right\}+R+\rho^{\prime}(0)(R-c y)\right)\right] .
\end{aligned}
$$

Taking the partial derivative with respect to $y$ and letting $y=R / c$, we obtain

$$
\begin{aligned}
& \frac{\partial \pi_{n}^{b}(R / c, R)}{\partial y} \\
& =\mathrm{E}\left[\left((p-c) 1_{\left\{R / c \leq D_{n}\right\}}-\rho^{\prime}(0) c\right) H_{n+1}^{\prime}\left((p-c) \min \left\{R / c, D_{n}\right\}+R\right)\right] \\
& \leq \mathrm{E}\left[(p-c) 1_{\left\{R / c \leq D_{n}\right\}}-\rho^{\prime}(0) c\right] \mathrm{E}\left[H_{n+1}^{\prime}\left((p-c) \min \left\{R / c, D_{n}\right\}+R\right)\right] \\
& =\left((p-c) \bar{F}(R / c)-\rho^{\prime}(0) c\right) \mathrm{E}\left[H_{n+1}^{\prime}\left((p-c) \min \left\{R / c, D_{n}\right\}+R\right)\right] \\
& \leq 0,
\end{aligned}
$$

where the first inequality is from the concavity of $H_{n+1}(\cdot)$; and the last one holds as $R \geq \mathrm{ca}_{n}^{\mathrm{br}}$ and $H_{n+1}(\cdot)$ is an increasing function.

Summarizing the above arguments, we have proved that (i) holds for $n$.

Second, we prove (ii) holds for $n$. When $R \geq c x$, by part (i) for $n$ and (13),

$$
\begin{aligned}
\tilde{V}_{n}(x, R) & =\pi_{n}\left(\max \left\{y_{n}^{*}(R), x\right\}, R\right) \\
& = \begin{cases}\pi_{n}(R / c, R), & \mathrm{ca}_{n}^{\mathrm{br}} \leq R \leq \mathrm{ca}_{n}^{d} ; \\
(1+d)^{N+1-n} R+G_{n}^{d}\left(\max \left\{a_{n}^{d}, x\right\}\right), & R \geq \mathrm{ca}_{n}^{d} .\end{cases}
\end{aligned}
$$

Thus, (ii) holds for $n$

Finally, we prove (iii) for $n$. When $R \geq \mathrm{ca}_{n+1}^{d}$, since (ii) holds for $n$ and $n+1$ and $H_{n}(\cdot)$ is a concave function, then $H_{n}^{\prime}(R) \geq(1+d)^{N-n+1}=$ $(1+d) H_{n+1}^{\prime}(R)$.

Now suppose $\mathrm{ca}_{n}^{\mathrm{br}} \leq R \leq \mathrm{ca}_{n+1}^{d}$. In this case, by (ii) for $n$ and (14), we obtain

$$
\begin{aligned}
H_{n}^{\prime}(R) & =\frac{\mathbf{d} \pi_{n}(R / c, R)}{\mathbf{d} R}=\frac{\partial \mathrm{E}\left[H_{n+1}\left((p-c) \min \left\{R / c, D_{n}\right\}+R\right)\right]}{\partial R} \\
& =\mathrm{E}\left[\left(\frac{p-c}{c} 1_{\left\{R / c \leq D_{n}\right\}}+1\right) H_{n+1}^{\prime}\left((p-c) \min \left\{R / c, D_{n}\right\}+R\right)\right] ;
\end{aligned}
$$

while by the inductive assumptions on (ii) for $n+1$ and $n+2$, we have

$$
\begin{aligned}
H_{n+1}^{\prime}(R)= & \frac{\mathbf{d} \pi_{n+1}(R / c, R)}{\mathbf{d} R} \\
= & \frac{\partial \mathrm{E}\left[\tilde{V}_{n+2}\left(\left(R / c-D_{n+1}\right)^{+},(p-c) \min \left\{R / c, D_{n+1}\right\}+R\right)\right]}{\partial R} \\
\leq & \mathrm{E}\left[\left(\frac{p-c}{c} 1_{\left\{R / c \leq D_{n+1}\right\}}+1\right)\right. \\
& \left.\times H_{n+2}^{\prime}\left((p-c) \min \left\{R / c, D_{n+1}\right\}+R\right)\right],
\end{aligned}
$$

where the inequality is from that $G_{n+2}\left(\max \left\{a_{n+2}^{d}, x\right\}\right)$ is decreasing in $x$.

Since $D_{n}$ and $D_{n+1}$ are identically distributed, it follows from the inductive assumption on (iii) that $H_{n}^{\prime}(R) \geq(1+d) H_{n+1}^{\prime}(R)$. Thus, (iii) holds for $n$. The proof is complete.

PROOF OF PROPOSITION 2: We first prove $y_{N}^{*}(R)=\arg \max _{y \geq R^{+} / c}$ $\pi_{N}(y, R)$ when $\underline{w}<R<\mathrm{ca}_{N}^{\mathrm{br}}$. From the definition of $y_{N}^{*}(R)$, it suffices to prove that $\pi_{N}(y, R)$ is increasing in $y$ on $0 \leq y \leq R / c$ and $0 \leq R<\mathrm{ca}_{N}^{\mathrm{br}}$. 
From the proof of Theorem 2, $\pi_{N}(y, R)=(1+d) R+G_{N}^{d}(y)$ when $0 \leq y \leq R / c$. By Lemma 2 , we have $a_{N}^{\mathrm{br}} \leq a_{N}^{d}$, hence $\pi_{N}(y, R)$ is increasing in $y$ on $0 \leq y \leq R / c<a_{N}^{\text {br }} \leq a_{N}^{d}$. Thus, the desired result holds.

Now suppose $y \geq R^{+} / c$. Since $\tilde{V}_{N+1}(x, R)=R+(\gamma-c) x$, it follows from (6) that

$$
\pi_{N}(y, R)=(p-c) y-(p-\gamma) \mathrm{E}\left[\left(y-D_{N}\right)^{+}\right]-\rho(c y-R)+R .
$$

Taking the partial derivative of $\pi_{N}(y, R)$ with respect to $y$, we obtain

$$
\frac{\partial \pi_{N}(y, R)}{\partial y}=p-c\left(1+\rho^{\prime}(c y-R)\right)-(p-\gamma) F(y) .
$$

Since $\pi_{N}(y, R)$ is concave in $y$, it is increasing in $y$ when $\frac{\partial \pi_{N}(y, R)}{\partial y} \geq$ 0 , or equivalently, when $F(y) \leq \frac{p-c\left(1+\rho^{\prime}(c y-R)\right)}{p-\gamma}$. Since $y_{N}^{*}(R)=$ $\arg \max _{y \geq R^{+} / c} \pi_{N}(y, R)$, we obtain

$$
y_{N}^{*}(R)=\sup \left\{y \geq R^{+} / c \mid F(y) \leq \frac{p-c\left(1+\rho^{\prime}(c y-R)\right)}{p-\gamma}\right\} .
$$

Since $\rho(\cdot)$ is a convex function, $\rho^{\prime}(c y-R)$ is decreasing in $R$, and $\frac{p-c\left(1+\rho^{\prime}(c y-R)\right)}{p-\gamma}$ is increasing in $R$ when $R \leq c y$. Thus, it directly follows from the definition of $y_{N}^{*}(R)$ that it is increasing in $R$. The proof is complete.

PROOF OF COROLLARY 2: For convenience, denote $\psi(R)$ as the righthand-side function of $R$ in the corollary, and we need to prove $y_{N}^{*}(R)=$ $\psi(R)$ when $-K_{m}<R<\mathrm{ca}_{N}^{b_{1}}$. By Proposition 2, it suffices to prove 1) when $R^{+} / c \leq y<\psi(R), F(y) \leq \frac{p-c\left(1+\rho^{\prime}(c y-R)\right)}{p-\gamma}$; and 2) when $y>\psi(R)$, $F(y)>\frac{p-c\left(1+\rho^{\prime}(c y-R)\right)}{p-\gamma}$. In what follows, we verify them sequentially.

We first verify (1). Suppose $R^{+} / c \leq y<\psi(R)$. Then, by Proposition 2, we have

$F(y)<F(\psi(R)) \leq\left\{\begin{array}{cc}F\left(a_{N}^{b_{m}}\right), & -K_{m}<R<\mathrm{ca}_{N}^{b_{m}}-K_{m} ; \\ F\left(a_{N}^{b_{m}}\right), & \mathrm{ca}_{N}^{b_{m}}-K_{m} \leq R<\mathrm{ca}_{N}^{b_{m}}-K_{m-1} ; \\ F\left(a_{N}^{b_{m-1}}\right), & \mathrm{ca}_{N}^{b_{m}}-K_{m-1} \leq R<\mathrm{ca}_{N}^{b_{m-1}}-K_{m-1} ; \\ \vdots & \vdots \\ F\left(a_{N}^{b_{1}}\right), & \mathrm{ca}_{N}^{b_{1}}-K_{1} \leq R<\mathrm{ca}_{N}^{b_{1}} .\end{array}\right.$

In addition, since $c y-R<c \psi(R)-R$ and by the definition of $\rho(z)$, we have

$$
\rho^{\prime}(c y-R) \leq\left\{\begin{array}{cc}
b_{m}, & -K_{m}<R<\mathrm{ca}_{N}^{b_{m}}-K_{m} \\
b_{m}, & \mathrm{ca}_{N}^{b_{m}}-K_{m} \leq R<\mathrm{ca}_{N}^{b_{m}}-K_{m-1} \\
b_{m-1}, & \mathrm{ca}_{N}^{b_{m}}-K_{m-1} \leq R<\mathrm{ca}_{N-1}^{b_{m-1}}-K_{m-1} \\
\vdots & \vdots \\
b_{1}, & \mathrm{ca}_{N}^{b_{1}}-K_{1} \leq R<\mathrm{ca}_{N}^{b_{1}}
\end{array}\right.
$$

Since $\left.F\left(a_{N}^{b_{i}}\right)=\frac{p-c\left(1+b_{i}\right)}{p-\gamma}, i=1, \ldots, m, 1\right)$ follows directly from the above two expressions.

We next verify (2). Suppose $y>\psi(R)$. Then, by Proposition 2, we have

$F(y)>F(\psi(R)) \geq\left\{\begin{array}{cc}F(0), & -K_{m}<R<\mathrm{ca}_{N}^{b_{m}}-K_{m} ; \\ F\left(a_{N}^{b_{m}}\right), & \mathrm{ca}_{N}^{b_{m}}-K_{m} \leq R<\mathrm{ca}_{N}^{b_{m}}-K_{m-1} ; \\ F\left(a_{N}^{b_{m}}\right), & \mathrm{ca}_{N}^{b_{m}}-K_{m-1} \leq R<\mathrm{ca}_{N}^{b_{m-1}}-K_{m-1} \\ \vdots & \vdots \\ F\left(a_{N}^{b_{1}}\right), & \mathrm{ca}_{N}^{b_{1}}-K_{1} \leq R<\mathrm{ca}_{N}^{b_{1}} .\end{array} ;\right.$

Naval Research Logistics DOI 10.1002/nav
In addition, since $c y-R>c \psi(R)-R$ and by the definition of $\rho(z)$, we have

$$
\rho^{\prime}(c y-R) \geq\left\{\begin{array}{cc}
(p-c) / c, & -K_{m}<R<\mathrm{ca}_{N}^{b_{m}}-K_{m} ; \\
b_{m}, & \mathrm{ca}_{N}^{b_{m}}-K_{m} \leq R<\mathrm{ca}_{N}-K_{m-1} ; \\
b_{m}, & \mathrm{ca}_{N}^{b_{m}}-K_{m-1} \leq R<\mathrm{ca}_{N}^{b_{m-1}}-K_{m-1} \\
\vdots & \vdots \\
b_{1}, & \mathrm{ca}_{N}^{b_{1}}-K_{1} \leq R<\mathrm{ca}_{N}^{b_{1}} .
\end{array}\right.
$$

Since $F\left(a_{N}^{b_{i}}\right)=\frac{p-c\left(1+b_{i}\right)}{p-\gamma}, i=1, \ldots, m,(2)$ follows directly from the above two expressions. The proof is complete.

PROOF OF PROPOSITION 3: As $y_{n}^{*}(R) \geq 0$, the proposition is trivially true when $R \leq 0$. Thus, we only prove $y_{n}^{*}(R) \geq R / c$ when $0<R \leq \mathrm{ca}_{n}^{\text {br }}$. In what follows, we suppose $0<R \leq \mathrm{ca}_{n}^{\mathrm{br}}$. When $n=N, y_{N}^{*}(R)$ is given in (9) and the result is clearly true. Thus, to complete the proof, by the definition of $y_{n}^{*}(R)$, it remains to prove that: (1) $\pi_{N-1}(y, R)$ is increasing in $y$ when $0 \leq y \leq R / c$; and (2) when $d=0, \pi_{n}(y, R)$ is increasing in $y$ when $0 \leq y \leq R / c, n=1, \ldots, N$.

When $0 \leq y \leq R / c$, by (6), we obtain

$\pi_{n}(y, R)=\mathrm{E}\left[\tilde{V}_{n+1}\left(\left(y-D_{n}\right)^{+},(p-c) \min \left\{y, D_{n}\right\}+(1+d) R-d\right.\right.$ cy $\left.)\right]$.

We first prove that $\pi_{N-1}(y, R)$ is increasing in $y$ when $0 \leq y \leq R / c$. When $\mathrm{ca}_{N}^{d} \leq R \leq \mathrm{ca}_{N-1}^{\mathrm{br}}$, by the proof of Theorem 2 , for any $0 \leq y \leq R / c$, we have

$$
\pi_{N-1}(y, R)=(1+d)^{2} R+G_{N-1}^{d}(y) .
$$

Thus, by the definition of $a_{N-1}^{d}$ and $a_{N-1}^{d} \geq a_{N-1}^{\text {br }}$ by Lemma $2, \pi_{N-1}(y, R)$ is increasing in $y$ when $0 \leq y \leq R / c$. In the following, we prove the desired result when $0<R \leq \mathrm{ca}_{N}^{d}$.

By Theorem 2 and (9), it is easy to verify that, when $R \geq c x, \tilde{V}_{N}(x, R)=$ $G_{N}^{d}\left(\max \left\{a_{N}^{d}, x\right\}\right)+H_{N}(R)$, where

$$
H_{N}(R)=\left\{\begin{array}{cc}
\pi_{n}\left(y_{N}^{*}(R), R\right)-G_{N}^{d}\left(a_{N}^{d}\right), & 0<R \leq \mathrm{ca}_{N}^{d} \\
(1+d) R, & R \geq \mathrm{ca}_{N}^{d}
\end{array}\right.
$$

Then, by Lemma 1 and Theorem 2 (ii), $H_{N}(R)$ is concave in $R$ and $H_{N}^{\prime}(R) \geq 1+d$. Thus, when $0<R<\mathrm{ca}_{N}^{d}$ and $y \leq R / c$, (17) can be rewritten as

$\pi_{N-1}(y, R)=G_{N}^{d}\left(a_{N}^{d}\right)+\mathrm{E}\left[H_{N}\left((p-c) \min \left\{y, D_{N-1}\right\}+(1+d) R-d c y\right)\right]$.

Taking the partial derivative with respect to $y$ and letting $y=R / c$, we obtain

$$
\begin{aligned}
\frac{\partial \pi_{N-1}(R / c, R)}{\partial y}= & (p-(1+d) c) \bar{F}(R / c) H_{N}^{\prime}(p R / c)-d c \\
& \mathrm{E}\left[1_{\left\{R / c \geq D_{N-1}\right\}} H_{N}^{\prime}\left((p-c) D_{N-1}+R\right)\right] \\
\geq & (p-(1+d) c)(1+d) \bar{F}(R / c)-d c F(R / c) H_{N}^{\prime}(R) \\
\geq & c d F(R / c)\left(\frac{(1+d)(p-c)}{c d} \bar{F}(R / c)-H_{N}^{\prime}(R)\right) \\
\geq & c d F(R / c)\left(1+\frac{p-c}{c} \bar{F}(R / c)-H_{N}^{\prime}(R)\right)
\end{aligned}
$$

where the first inequality is from the concavity of $H_{N}(\cdot)$ and $H_{N}^{\prime}(R) \geq$ $1+d$, and the remaining two inequalities are both from $R / c \leq a_{N}^{d} \leq$ $F^{-1}\left(\frac{p-(1+d) c}{p-c}\right)$. 
Since $\pi_{N-1}(y, R)$ is concave in $y$, to prove the desired result, it suffices to show $\frac{\partial \pi_{N-1}(R / c, R)}{\partial y} \geq 0$. By (18), it is sufficient to prove that, when $0<R \leq \mathrm{ca}_{N}^{d}$,

$$
H_{N}^{\prime}(R) \leq 1+\frac{p-c}{c} \bar{F}(R / c) .
$$

Note that $y_{N}^{*}(R) \geq R / c$ when $0<R \leq \mathrm{ca}_{N}^{d}$. In what follows, we divide the analysis into the following two cases and separately prove (19) holds in both cases.

CASE 1: $y_{N}^{*}(R)=R / c$. In this case, by (6), we obtain

$$
\begin{aligned}
H_{N}^{\prime}(R) & =\frac{\mathbf{d} \pi_{N}(R / c, R)}{\mathbf{d} R}=1+\frac{p-c}{c} \bar{F}(R / c)+\frac{\gamma-c}{c} F(R / c) \\
& \leq 1+\frac{p-c}{c} \bar{F}(R / c)
\end{aligned}
$$

where the inequality follows from $c \geq \gamma$. Hence, (19) holds when $y_{N}^{*}(R)=$ $R / c$.

CASE 2: $y_{N}^{*}(R)>R / c$. In this case, for any $y>R / c$, by (6), we obtain

$$
\begin{array}{r}
\pi_{N}(y, R)=(\gamma-c) \mathrm{E}\left[\left(y-D_{N}\right)^{+}\right]+(p-c) \\
\mathrm{E}\left[\min \left\{y, D_{N}\right\}\right]-\rho(c y-R)+R .
\end{array}
$$

Taking the partial derivative with respect to $y$ and letting $y=y_{N}^{*}(R)$, we obtain

$$
\frac{\partial \pi_{N}\left(y_{N}^{*}(R), R\right)}{\partial y}=(\gamma-c) F\left(y_{N}^{*}(R)\right)+(p-c) \bar{F}\left(y_{N}^{*}(R)\right)-c \rho^{\prime}\left(c y_{N}^{*}(R)-R\right) .
$$

Since $y_{N}^{*}(R)>R / c, \frac{\partial \pi_{N}\left(y_{N}^{*}(R), R\right)}{\partial y}=0$. In addition, since $\gamma \leq c$, it follows that

$$
c \rho^{\prime}\left(c y_{N}^{*}(R)-R\right) \leq(p-c) \bar{F}\left(y_{N}^{*}(R)\right) \leq(p-c) \bar{F}(R / c) .
$$

By the definition of $\tilde{H}_{N}(R)$, we have

$$
\begin{aligned}
H_{N}^{\prime}(R) & =\frac{\mathbf{d} \pi_{N}\left(y_{N}^{*}(R), R\right)}{\mathbf{d} R} \\
& =1+\rho^{\prime}\left(c y_{n+1}^{*}(R)-R\right) \leq 1+\frac{p-c}{c} \bar{F}(R / c) .
\end{aligned}
$$

Thus, (19) also holds when $y_{N}^{*}(R)>R / c$.

Therefore, we have shown $\frac{\partial \pi_{N-1}(R / c, R)}{\partial y} \geq 0$ when $0<R \leq \mathrm{ca}_{N}^{d}$. The proof for (1) is complete.

We next prove that, when $d=0, \pi_{n}(y, R)$ is increasing in $y$ when $0 \leq y \leq R / c, n=1, \ldots, N$. When $\mathrm{ca}_{n+1}^{d} \leq R \leq \mathrm{ca}_{n}^{\mathrm{br}}$, by the proof of Theorem 2 , for any $0 \leq y \leq R / c$, we have

$$
\pi_{n}(y, R)=R+G_{n}^{d}(y) .
$$

Thus, by the definition of $a_{n}^{d}$ and $a_{n}^{d} \geq a_{n}^{\text {br }}$ by Lemma $2, \pi_{n}(y, R)$ is increasing in $y$ when $0 \leq y \leq R / c$.

In what follows, we prove by induction on $n$ that $y_{n}^{*}(R) \geq R / c$ when $0<R \leq \mathrm{ca}_{n+1}^{d}$. From the first part of the proof, the result is obviously true for $n=N$. Now assume inductively that the result is true for $n+1$. To complete the proof, we shall prove that the result is also true for $n$.
By Theorem 2 and the inductive assumption, it is easy to verify that, when $R \geq c x, \tilde{V}_{n+1}(x, R)=G_{n+1}^{d}\left(\max \left\{a_{n+1}^{d}, x\right\}\right)+H_{n+1}(R)$, where

$$
H_{n+1}(R)= \begin{cases}\pi_{n}\left(y_{n+1}^{*}(R), R\right)-G_{n+1}^{d}\left(a_{n+1}^{d}\right), & 0<R \leq \mathrm{ca}_{n+1}^{d} ; \\ R, & R \geq \mathrm{ca}_{n+1}^{d} .\end{cases}
$$

Thus, when $0<R \leq \mathrm{ca}_{n+1}^{d}$ and $y \leq R / c$, (17) can be simplified as

$$
\pi_{n}(y, R)=G_{n+1}^{d}\left(a_{n+1}^{d}\right)+\mathrm{E}\left[H_{n+1}\left((p-c) \min \left\{y, D_{n}\right\}+R\right)\right] .
$$

Note that $H_{n+1}(\cdot)$ is an increasing function. Thus, $\pi_{n}(y, R)$ is increasing in $y$ when $y \leq R / c$. Hence, $y_{n}^{*}(R) \geq R / c$ when $0<R \leq \mathrm{ca}_{n+1}^{d}$. The proof is complete.

PROOF OF LEMMA 3: Notice that $a_{n}^{b}$ is a special case of $a_{n}^{d}$ when $d=b$. Thus, part (i) directly follows from Lemma 2 (i). We now prove part (ii). First, by definition and part (i), $a_{N}^{\mathrm{bl}}=a_{N}^{b}=F^{-1}\left(\frac{p-(1+b) c}{p-\gamma}\right)$.

Second, we prove by induction on $n$ that $a_{n+1}^{\text {bl }}>a_{n}^{\text {bl }}, n=1, \ldots, N-1$. When $n=N-1$, it follows from (10) that

$$
a_{N-1}^{\mathrm{bl}}=\frac{\mathrm{ca}_{N}^{b}-(p-(1+b) c) a_{N-1}^{b}}{(1+b) c} \leq \frac{1}{1+b} a_{N}^{b}<a_{N}^{b}=a_{N}^{\mathrm{bl}} .
$$

Thus, $a_{N}^{\mathrm{bl}}>a_{N-1}^{\mathrm{bl}}$. Now assume inductively that $a_{n+1}^{\mathrm{bl}}>a_{n}^{\mathrm{bl}}$. By the definition of $a_{n}^{\text {bl }}$ in (10),

$$
a_{n}^{\mathrm{bl}}-a_{n-1}^{\mathrm{bl}}=\frac{c\left(a_{n+1}^{\mathrm{bl}}-a_{n}^{\mathrm{bl}}\right)-(p-(1+b) c)\left(a_{n}^{b}-a_{n-1}^{b}\right)}{(1+b) c} .
$$

Since $a_{n+1}^{\mathrm{bl}}>a_{n}^{\mathrm{bl}}$ by the inductive assumption and $a_{n}^{b} \leq a_{n-1}^{b}$ from part (i), we obtain $a_{n}^{\mathrm{bl}}>a_{n-1}^{\mathrm{bl}}$. Thus, by induction, we have shown that $a_{n+1}^{\mathrm{bl}}>a_{n}^{\mathrm{bl}}$, $n=1, \ldots, N-1$.

Finally, we prove $a_{1}^{\mathrm{bl}}>-\frac{p-(1+b) c}{b c} F^{-1}\left(\frac{p-(1+b) c}{p-c}\right)$. By the definition of $a_{1}^{\mathrm{bl}}$ and $a_{2}^{\mathrm{bl}}>a_{1}^{\mathrm{bl}}$, we have $a_{1}^{\mathrm{bl}}=\frac{c a_{2}^{\mathrm{bl}}-(p-(1+b) c) a_{1}^{b}}{(1+b) c}>\frac{\mathrm{ca}{ }_{1}^{\mathrm{bl}}-(p-(1+b) c) a_{1}^{b}}{(1+b) c}$. Then, it follows that

$$
a_{1}^{\mathrm{bl}}>-\frac{p-(1+b) c}{b c} a_{1}^{b} \geq-\frac{p-(1+b) c}{b c} F^{-1}\left(\frac{p-(1+b) c}{p-c}\right),
$$

where the second inequality is from part (i). The proof is complete.

PROOF OF THEOREM 3: The proof is by induction on $n$. When $R \leq$ $\mathrm{ca}_{N}^{\mathrm{bl}}=\mathrm{ca}_{N}^{\mathrm{br}}$, it directly follows from Proposition 2 that $y_{N}^{*}(R)=a_{N}^{b}$. In addition, since $\tilde{V}_{N+1}(x, R)=R+(\gamma-c) x$, we have $\pi_{N}(y, R)=$ $(1+b) R+G_{N}^{b}(y)$ when $y \geq R^{+} / c$. Thus, when $R \leq \mathrm{ca}_{N}^{\mathrm{bl}}$ and $x \geq 0$,

$$
\begin{aligned}
\tilde{V}_{N}(x, R) & =\max _{y \geq x} \pi_{N}(y, R)=\pi_{N}\left(\max \left\{a_{N}^{b}, x\right\}, R\right) \\
& =(1+b) R+G_{N}^{b}\left(\max \left\{a_{N}^{b}, x\right\}\right) .
\end{aligned}
$$

This proves the result for $N$. Now assume inductively that the theorem holds for $n+1$. In what follows, we prove that it also holds for $n$, which then completes the proof.

Suppose $R \leq \mathrm{ca}_{n}^{\mathrm{bl}}$. Then, by the definition of $a_{n}^{\mathrm{bl}}$, we have $R \leq$ $\frac{\mathrm{ca}_{n+1}^{\mathrm{bl}}-(p-(1+b) c) a_{n}^{b}}{1+b}$, or equivalently, $a_{n}^{b} \leq \frac{c a_{n+1}^{\mathrm{bl}}-(1+b) R}{p-(1+b) c}$. Thus, by Lemma 3, we have $R / c \leq a_{n}^{\mathrm{bl}} \leq a_{n}^{b} \leq \frac{\mathrm{ca}_{n+1}^{\mathrm{bl}}-(1+b) R}{p-(1+b) c}$.

When $0 \leq y \leq \frac{\mathrm{ca}_{n+1}^{\mathrm{bl}}-(1+b) R}{p-(1+b) c}$, for all $D_{n} \geq 0$, we have

$$
\begin{aligned}
(p-c) \min \left\{y, D_{n}\right\}+(1+b) R- & b c y \\
& \leq(p-(1+b) c) y+(1+b) R \leq \mathrm{ca}_{n+1}^{\mathrm{bl}} ;
\end{aligned}
$$

Naval Research Logistics DOI 10.1002/nav 
and, since $a_{n+1}^{\mathrm{bl}} \leq a_{n+2}^{\mathrm{bl}} /(1+b)$ from the definition of $a_{n}^{\mathrm{bl}}$, we also have

$$
\begin{aligned}
\left(y-D_{n}\right)^{+} \leq & \frac{(1+b)(p-c)}{p-(1+b) c}\left(y-D_{n}\right)^{+} \\
& +\frac{\mathrm{ca}_{n+2}^{\mathrm{bl}}-(1+b)((p-(1+b) c) y+(1+b) R)}{p-(1+b) c} \\
= & \frac{\mathrm{ca}_{n+2}^{\mathrm{bl}}-(1+b)\left((p-c) \min \left\{y, D_{n}\right\}+(1+b) R-b c y\right)}{p-(1+b) c} .
\end{aligned}
$$

Thus, when $R^{+} / c \leq y \leq \frac{\mathrm{ca}_{n+1}^{\mathrm{bl}}-(1+b) R}{p-(1+b) c}$, by the inductive assumption on (ii) for $n+1$, we have

$$
\begin{aligned}
\pi_{n}(y, R)= & \mathrm{E}\left[\tilde{V}_{n+1}\left(\left(y-D_{n}\right)^{+},(p-c) \min \left\{y, D_{n}\right\}+(1+b) R-b c y\right)\right] \\
= & \mathrm{E}\left[G_{n+1}^{b}\left(\max \left\{a_{n+1}^{b},\left(y-D_{n}\right)^{+}\right\}\right)\right] \\
& +(1+b)^{N-n}\left((p-c) \mathrm{E}\left[\min \left\{y, D_{n}\right\}\right]+(1+b) R-b c y\right) \\
= & G_{n}^{b}(y)+(1+b)^{N-n+1} R .
\end{aligned}
$$

Since $a_{n}^{b}$ is the maximizer of $G_{n}^{b}(y), \pi_{n}(y, R)$ is increasing in $y$ when $R^{+} / c \leq y \leq a_{n}^{b}$ and it is decreasing in $y$ when $a_{n}^{b} \leq y \leq \frac{\mathrm{ca}_{n+1}^{\mathrm{bl}}-(1+b) R}{p-(1+b) c}$. Since $\pi_{n}(y, R)$ is concave in $y$ when $y \geq 0$, we conclude that $y_{n}^{*}(R)=a_{n}^{b}$ when $R \leq \mathrm{ca}_{n}^{\text {bl }}$. Thus, (i) holds for $n$.

When $R \leq \mathrm{ca}_{n}^{\mathrm{bl}}$ and $0 \leq x \leq \frac{\mathrm{ca}_{n+1}^{\mathrm{bl}}-(1+b) R}{p-(1+b) c}$, since $R / c \leq a_{n}^{b} \leq$ $\frac{\mathrm{ca}_{n+1}^{\mathrm{bl}}-(1+b) R}{p-(1+b) c}$, we have $R^{+} / c \leq \max \left\{a_{n}^{b}, x\right\} \leq \frac{\mathrm{ca}_{n+1}^{\mathrm{bl}}-(1+b) R}{p-(1+b) c}$. Then, by applying the above expression for $\pi_{n}(y, R)$, we obtain

$$
\begin{aligned}
\tilde{V}_{n}(x, R) & =\max _{y \geq x} \pi_{n}(y, R)=\pi_{n}\left(\max \left\{a_{n}^{b}, x\right\}, R\right) \\
& =G_{n}^{b}\left(\max \left\{a_{n}^{b}, x\right\}\right)+(1+b)^{N-n+1} R .
\end{aligned}
$$

This shows that (ii) holds for $n$. Thus, the result has been shown to hold for an arbitrary $n$, and the proof is complete.

PROOF OF THEOREM 4: The proofs for the results in Theorem 4 are similar to those for the corresponding results in Theorems 2 and 3. Hence, they are omitted here for brevity.

PROOF OF PROPOSITION 4: When $w+c x \geq \mathrm{ca}_{N}^{\mathrm{br}}$ or $w+c x \leq \underline{w}$, from Theorems 1 and 2 and Corollary 1, we know that it is optimal for the firm not to borrow in period $N$ when $K=0$. In this case, it is clear that it is optimal for the firm not to borrow either when $K>0$. Consequently, when $w+c x \geq \mathrm{ca}_{N}^{\mathrm{br}}$ or $w+c x \leq \underline{w}$, from Theorems 1 and 2 and Corollary 1 one can easily verify that the firm's optimal policy is correctly given in Proposition 4.

Now we suppose $\underline{w}<w+c x<\mathrm{ca}_{N}^{\text {br }}$. In this case, we know from Proposition 2 that it is optimal for the firm to borrow some capital and raise its inventory up to the level $y_{N}^{*}(w+c x)$ if $K=0$. Note that the firm's objective function is concave in $y$ when $K=0$. Then, for the region when the firm does not borrow, it is optimal for the firm to use up its own capital $w^{+}$to purchase inventory. On the other hand, for the region when the firm borrows, since the fixed cost $K$ becomes a sunk cost, it is optimal for the firm to raise its inventory up to level $y_{N}^{*}(w+c x)$. Consequently, the firm's optimal decision is the one of the above two decisions that gives higher expected terminal wealth.

Finally, when $\rho(z)=b z$ with $d \leq b<(p-c) / c$, we have $\underline{w}=-\infty$ and $y_{N}^{*}(w+c x)=a_{N}^{b}$ when $w+c x \leq \mathrm{ca}_{N}^{\text {br }}$. In addition, when $w \geq 0$, it is easy to verify that the expected terminal wealth when the firm uses up its capital $w$ to purchase inventory is $G_{N}^{b}(w / c+x)+(1+b)(w+c x)$; whereas the expected terminal wealth when the firm orders its inventory up to $a_{N}^{b}$ is $G_{N}^{b}\left(a_{N}^{b}\right)+(1+b)(w+c x)-K$. Note that $G_{N}^{b}(y)$ is a concave function and achieves maximization when $y=a_{N}^{b}$. Thus,

$$
\begin{array}{r}
G_{N}^{b}(w / c+x)+(1+b)(w+c x)-\left(G_{N}^{b}\left(a_{N}^{b}\right)+(1+b)(w+c x)-K\right) \\
=G_{N}^{b}(w / c+x)-G_{N}^{b}\left(a_{N}^{b}\right)+K
\end{array}
$$

is increasing in $w+c x$ and it is positive when $w+c x=a_{N}^{b}$. Therefore, there exists a critical point $r^{*}$ (which can be $-\infty$ ) such that it is optimal to order up to $a_{N}^{b}$ when $w+c x \leq r^{*}$ and to use up the firm's capital $w$ when $r^{*}<w+c x<\mathrm{ca}_{N}^{b}$. The proof is complete.

\section{ACKNOWLEDGMENTS}

The authors are grateful to the Editor-in-Chief Awi Federgruen, the associate editor, and three anonymous referees for their detailed comments and suggestions, which have helped to significantly improve both the content and the exposition of this paper. The authors thank Dr. Jia Chen of the Bank of China for helpful discussions during the early stage of this paper. This research is supported in part by CUHK Direct Grant under No. 4055022 for X. Gong and by the National Science Foundation (Grants CMMI-0927631 and CMMI-1131249 for X. Chao and Grant CMMI-0758069 for D. Simchi-Levi).

\section{REFERENCES}

[1] T. Archibald, L. Thomas, J. Betts, and R. Johnston, Should start-up companies be cautious? Inventory policies which maximize survival probabilities, Manag Sci 4 (2002), 1161-1174.

[2] V. Babich, G. Aydin, P.-Y. Brunet, J. Keppo, and R. Saigal, "Risk, financing and the optimal number of suppliers," Supply chain disruptions: Theory and practice of managing risk, $\mathrm{H}$. Gurnani, A. Mehrotra, and S. Ray (Editors), Springer-Verlag London Ltd., London, UK, 2012.

[3] V. Babich and M.J. Sobel, Pre-IPO operational and financial decisions, Manag Sci 50 (2004), 935-948.

[4] J.A. Buzacott and R.Q. Zhang, Inventory management with asset-based financing, Manag Sci 50 (2004), 1274-1292.

[5] X. Chao, J. Chen, and S. Wang, Dynamic inventory management with cash flow constraints, Nav Res Log 55 (2008), $758-768$.

[6] M. Dada and Q. Hu, Financing newsvendor inventory, Oper Res Lett 36 (2008), 569-573.

[7] L. Eeckhoudt, C. Gollier, and H. Schlesinger, The risk-averse (and prudent) newsboy, Manag Sci 41 (1995), 786-794.

[8] D.P. Heyman and M.J. Sobel, Stochastic models in operations research, Vol. II: Stochastic optimization, McGraw-Hill, NY, 1984.

[9] Q. Hu, L. Li, and M.J. Sobel, "Production/inventory management and capital structure," The handbook of integrated risk management in global supply chains, P. Kouvelis, L. Dong, O. Boyabatli, and R. Li (Editors), John Wiley \& Sons, Inc, Hoboken, New Jersey, 2012.

[10] Q. Hu and M.J. Sobel, Echelon base-stock policies are financially sub-optimal, Oper Res Lett 35 (2007), 561-566. 
[11] Q. Hu, M.J. Sobel, and D. Turcic, "Optimization of inventory and dividends with risky debt", Working paper, Case Western Reserve University, Cleveland, OH, 2010.

[12] I. Karatzas, "Lectures on the mathmatics of finance", CRM Monograph Series, American Mathematical Society, Providence, RI, 1997.

[13] P. Kouvelis and W. Zhao, The newsvendor problem and priceonly contract when bankruptcy costs exist, Prod Oper Manag 20 (2011), 921-936.

[14] L. Li, M. Shubik, and M.J. Sobel, Control of dividends, capital subscriptions, and physical inventories, Manag Sci 59 (2013), 1107-1124.

[15] F. Modigliani and M.H. Miller, The cost of capital, corporation finance, and the theory of investment, Ame Econ Rev 48 (1958), 261-297.

[16] S.R. Pliska, Introduction to mathematical finance: discrete time models, Blackwell Publishers, Malden, MA, 1997.

[17] J. Shi, M.N. Katehakis, and B. Melamed, "Cash-flow based dynamic inventory management", Working paper, Rutgers Business School, Newark, NJ 07102, 2012.
[18] X. Xu and J.R. Birge, "Joint proudction and financing decisions: Modeling and analysis", Working paper, available at http://dx.doi.org/10.2139/ssrn.652562. Accessed on February 5, 2014, 2004.

[19] X. Xu and J.R. Birge, Equity valuation, production, and financial planning: A stochastic programming approach, Nav Res Log 53 (2006), 641-655.

[20] X. Xu and J.R. Birge, Operational decisions, capital structure, and managerial compensation: A news vendor perspective, Eng Econ 53 (2008), 173-196.

[21] S. Yang and J.R. Birge, "How inventory is (should be) financed: Trade credit in supply chains with demand uncertainty and costs of financial distress", Working paper, available at http://dx.doi.org/10.2139/ssrn.1734682. Accessed on February 5, 2014, 2011.

[22] E. Zakrajšek, "Retail inventories, internal finance, and aggregate fluctuations: Evidence from U.S. firm-level data", Research Paper No. 9722, Federal Reserve Bank of New York, New York, NY 10045, 1997.

[23] P.H. Zipkin, Foundations of inventory management, McGrawHill, Boston, MA, 2000. 